\title{
A Novel 2D Graphene Oxide Modified a-AgVO3 Nanorods: Design, Fabrication and Enhanced Visible-Light Photocatalytic Performance
}

Yan Xing ( $\sim$ xingyan618@njupt.edu.cn )

Nanjing University of Posts and Telecommunications https://orcid.org/0000-0002-7210-2458

Jian WU

Nanjing University of Posts and Telecommunications

Xin MIN

China University of Geosciences Beijing

Xingao LI

Nanjing University of Posts and Telecommunications

\section{Research Article}

Keywords: a-AgVO3, GO/a-AgVO3, Photocatalysis, GO nanosheets, In-suit coprecipitation processing

Posted Date: June 3rd, 2021

DOI: https://doi.org/10.21203/rs.3.rs-577266/v1

License: (a) (i) This work is licensed under a Creative Commons Attribution 4.0 International License. Read Full License 


\section{Title Page}

A novel 2D graphene oxide modified $\alpha$-AgVOs nanorods: Design, fabrication and enhanced visible-light photocatalytic performance

$$
\text { Jian WU }{ }^{\mathrm{a}} \text {, Yan XING }{ }^{\mathrm{a}^{*}}, \text { Xin MIN }{ }^{\mathrm{b}} \text {, Xingao LI }{ }^{\mathrm{a}^{*}}
$$

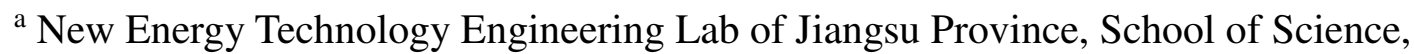

Nanjing University of Posts \& Telecommunications (NUPT), Nanjing 210023, China

${ }^{b}$ Beijing Key Laboratory of Materials Utilization of Nonmetallic Minerals and Solid Wastes, National Laboratory of Mineral Materials, School of Materials Science and Technology, China University of Geosciences (Beijing), Beijing 100083, China

Corresponding authors:

Dr. Yan Xing, E-mail: xingyan618@njupt.edu.cn

Dr. Xing’ao Li, E-mail: lixa@njupt.edu.cn 


\begin{abstract}
Silver vanadates are promising visible-light-responded photocatalysts with suitable bandgap for solar absorption. However, the easy recombination of photogenerated carriers limits their performance. To overcome this obstacle, a novel 2D graphene oxide (GO) modified $\alpha-\mathrm{AgVO}_{3}$ nanorods $\left(\mathrm{GO} / \alpha-\mathrm{AgVO}_{3}\right)$ photocatalyst was designed herein to improve the separation of photocarriers. The $\mathrm{GO} / \alpha-\mathrm{AgVO}_{3}$ was fabricated through a facile in-suit coprecipitation method at room temperature. It was found that the as-prepared $0.5 \mathrm{wt} . \% \mathrm{GO} / \alpha-\mathrm{AgVO}_{3}$ exhibited the most excellent performance for Rhodamine $\mathrm{B}(\mathrm{RhB})$ decomposition, with an apparent reaction rate constant 18 times higher than that of pure $\alpha-\mathrm{AgVO}_{3}$ under visible-light irradiation. In light of the first-principles calculations and the heterojunction analysis, the mechanism underpinned the enhanced photocatalytic performance was proposed. The enhanced photocatalytic performance was ascribed to the appropriate bandgap of $\alpha-\mathrm{AgVO}_{3}$ nanorods for visible light response and efficient separation of photocarriers through GO nanosheets. This work demonstrates the feasibility of overcoming the easy recombination of photogenerated carriers and provides a valuable $\mathrm{GO} / \alpha-\mathrm{AgVO}_{3}$ photocatalyst for pollutant degradation.
\end{abstract}

Keywords: $\alpha-\mathrm{AgVO}_{3} ; \mathrm{GO} / \alpha-\mathrm{AgVO}_{3} ;$ Photocatalysis; $\mathrm{GO}$ nanosheets; In-suit coprecipitation processing 


\section{Introduction}

The elimination of toxic organic dyes that hazarded to water resources and human health has aroused widespread attentions [1,2]. Photocatalysis has been regarded as a promising approach to surmount these concerns, wherein visible-light-driven photocatalysts play an important role $[3,4]$. However, currently, there are two limitations that hinder the performance and applications of photocatalysts. One is that the band gap of photocatalyst is not suitable for visible light absorption [5,6], the other is the rapid recombination of photogenerated electrons and holes [7]. To cope with these issues, it is appealing to search for novel photocatalysts with fascinating properties $[8,9]$.

Silver vanadium oxides (SVOs), including $\mathrm{AgVO}_{3}[10], \mathrm{Ag}_{4} \mathrm{~V}_{2} \mathrm{O}_{7}$ [11], $\mathrm{Ag}_{3} \mathrm{VO}_{4}$ [12], and $\mathrm{Ag}_{2} \mathrm{~V}_{4} \mathrm{O}_{11}$ [13], have been widely exploited in the field of catalysis owing to their excellent electrical properties, controllable surface, and easy availability. Among these SVOs, monoclinic structured $\alpha$ phase $\mathrm{AgVO}_{3}$ is one of the most promising visible-light-responsible photocatalyst because the hybridization of $\mathrm{V} 3 d, \mathrm{O} 2 p$, and $\mathrm{Ag} 4 d$ orbits can form highly dispersed valence bands with a narrow band gap [14]. For its potential high performance, photocatalytic properties of $\alpha-\mathrm{AgVO}_{3}$ based materials have been explored extensively $[15,16]$. However, the activity of pristine $\mathrm{AgVO}_{3}$ is still unsatisfactory owing to the fast recombination of photogenerated electrons and holes, which will reduce the photoreaction rate and thus limit its further applications.

Heterojunction construction is an efficient way to overcome the above obstacles.

By designing proper heterostructure, including matched band structure and 
well-controlled surface, an additional transmission path for the carriers (electrons and holes) can be established at the interface which is helpful to improve the photocatalytic performance. Considering the high chemical stability, large surface area, and high electron mobility, 2D graphene oxide (GO) is regarded as an ideal candidate to act as an electron transmitter [17,18]. For example, enhanced photocatalytic activity has been found in $\mathrm{GO} / \mathrm{g}-\mathrm{C}_{3} \mathrm{~N}_{4} / \mathrm{MoS}_{2}$ ternary hybrid material, wherein the fast charge transfer pathway through GO plays a key role [19]. Based on these facts, the introduction of $\mathrm{GO}$ to build $\mathrm{GO} / \alpha-\mathrm{AgVO}_{3}$ nanorods hybrid materials is expected to significantly improve the photocatalytic performance of $\alpha-\mathrm{AgVO}_{3}$.

With this aim, a series of $\mathrm{GO} / \alpha-\mathrm{AgVO}_{3}$ photocatalysts were designed in this work and were fabricated by a facial in-suit coprecipitation method. The structure and morphology of the as-prepared $\mathrm{GO} / \alpha-\mathrm{AgVO}_{3}$ were systematically characterized. And the photocatalytic performance of $\mathrm{GO} / \alpha-\mathrm{AgVO}_{3}$ was evaluated using photodecomposition of $\mathrm{RhB}$ as a model reaction. The dynamic of degradative reaction was also analyzed. Moreover, in order to disclose the possible mechanism that underpins the enhanced photocatalytic performance, the band structure of $\alpha-\mathrm{AgVO}_{3}$ was calculated by first-principles calculations based on DFT.

\section{Experimental Procedure}

\subsection{Preparation of $\alpha-\mathrm{AgVO}_{3}$ nanorods and $\mathrm{GO} / \alpha-\mathrm{AgOO}_{3}$ photocatalysts}

Ammonium metavanadate $\left(\mathrm{NH}_{4} \mathrm{VO}_{3}, \geq 99 \%\right.$, analytical reagent purchased from Aladdin Chemical Co., Ltd.), silver nitrate $\left(\mathrm{AgNO}_{3}, \geq 99 \%\right.$, analytical reagent) and Rhodamine B (RhB, purchased from Sinopharm Chemical Reagent Co Ltd.), and 
Graphene oxide (type SE2430 obtained from the Sixth Elemental Inc.) were used as starting materials. All the reagents were used without further purification.

Pure $\alpha-\mathrm{AgVO}_{3}$ nanorods were fabricated using a coprecipitation method at room

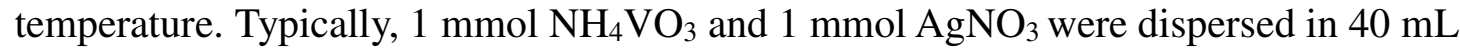
of deionized water, respectively, and stirred for $1 \mathrm{~h}$ to dissolve completely. The $\mathrm{AgNO}_{3}$ solution was added into the $\mathrm{NH}_{4} \mathrm{VO}_{3}$ solution drop wisely under agitation, then the mixture was stirred continuously for $3 \mathrm{~h}$ at room temperature. Then $\alpha-\mathrm{AgVO}_{3}$ nanorods were obtained by centrifugation at $6000 \mathrm{r} / \mathrm{min}$ for $10 \mathrm{~min}$. The nanorods were washed with distilled water and ethanol for three times and then dried in an oven at $40{ }^{\circ} \mathrm{C}$ overnight.

The $\mathrm{GO} / \alpha-\mathrm{AgVO}_{3}$ photocatalysts were fabricated by a facial in-situ directly coprecipitation method. Firstly, GO nanosheets $(20 \mathrm{mg})$ were added to deionized water $(100 \mathrm{~mL})$, and the suspension was sonicated for $4 \mathrm{~h}$ at $10{ }^{\circ} \mathrm{C}$. Likewise, $1 \mathrm{mmol}$ $\mathrm{NH}_{4} \mathrm{VO}_{3}$ and $1 \mathrm{mmol} \mathrm{AgNO}_{3}$ were prepared separately. The 2D GO nanosheets dispersion with a certain mass fraction ratio $(0.5 \mathrm{wt} . \%, 1.0 \mathrm{wt} . \%, 2.0 \mathrm{wt} . \%)$ was added to $\mathrm{NH}_{4} \mathrm{VO}_{3}$ solution and stirred for 30 min to reach saturated adsorption of $\mathrm{VO}_{3}{ }^{-}$on the surface of GO. Then, the $\mathrm{AgNO}_{3}$ solution was dropped into the mixture slowly, and the mixture was stirred continuously for $3 \mathrm{~h}$ at room temperature. The as-prepared $\mathrm{GO} / \alpha-\mathrm{AgVO}_{3}$ hybrid materials were obtained by centrifugation at $6000 \mathrm{r} / \mathrm{min}$ for 10 min. The hybrid materials were washed with distilled water and ethanol three times and then dried in an oven at $40{ }^{\circ} \mathrm{C}$. Briefly, the as-synthesized photocatalysts were 
referred as $0.5 \mathrm{GO} / \alpha-\mathrm{AgVO}_{3}, 1.0 \mathrm{GO} / \alpha-\mathrm{AgVO}_{3}$ and $2.0 \mathrm{GO} / \alpha-\mathrm{AgVO}_{3}$ according to the GO weight percentage, respectively.

\subsection{Structural characterization and photocatalytic activity}

The phase compositions of the $\alpha-\mathrm{AgVO}_{3}$ and $\mathrm{GO} / \alpha-\mathrm{AgVO}_{3}$ were characterized by X-ray diffraction (XRD) on a Bruker $\mathrm{D} 8$ diffractometer with $\mathrm{Cu} \mathrm{K} \alpha$ radiation $(\lambda=1.5418 \AA)$. The morphology characterizations were conducted on Hitachi S-4800 scanning electron microscope (SEM) with energy dispersive spectroscopy (EDS) for elemental analysis. UV-vis diffuse reflectance spectra (DRS) were carried out on a UV-vis spectrophotometer (UV-3600 PLUS) equipped with an integrating sphere device in the wavelength range of 300-800 $\mathrm{nm}$. Ultraviolet photoelectron spectroscopy (UPS) was characterized with He I $(21.22 \mathrm{eV})$ as the monochromatic light source and the total energy resolution is $100 \mathrm{meV}$. The $\mathrm{X}$-ray photoelectron spectroscopy (XPS) measurements were measured on ESCALAB 250Xi with an Al $\mathrm{K} \alpha$ source to identify surface chemical composition and chemical states.

The photocatalytic activities of the as-prepared $\alpha-\mathrm{AgVO}_{3}$ and $\mathrm{GO} / \alpha-\mathrm{AgVO}_{3}$ were evaluated via the photodegradation efficiency of Rhodamine $B(R h B)$. The experiments were carried out in a photoreactor containing a $300 \mathrm{~W}$ Xenon lamp (400 $\mathrm{nm}$ cutoff filter) and a circulating cooling water system. For the catalytic experiments, $15 \mathrm{mg}$ of the as-prepared samples were dispersed in $30 \mathrm{ml} \mathrm{RhB}$ solution $(5 \mathrm{mg} / \mathrm{L})$ and stirred for $30 \mathrm{~min}$ in dark to reach the adsorption-desorption equilibrium. Then, the light source was switched on to start the photoreaction, during which, the residual concentration of RhB was monitored by UV-vis absorption spectra at certain time 
intervals. The degradation efficiency was calculated from the absorbance of the dye solution at a wavelength of $552 \mathrm{~nm}$.

\subsection{Band structure calculation}

The band structure of $\alpha-\mathrm{AgVO}_{3}$ was investigated to understand its photocatalytic performance. For this aim, first-principles calculations were conducted using the Cambridge serial total energy package (CASTEP) code [20,21]. The plane-wave basis set cutoff was $760 \mathrm{eV}$ after convergent test. A $2 \times 2 \times 3$ Monckhorst-Pack $k$-mesh was used to sample the Brillouin zone of $\alpha-\mathrm{AgVO}_{3}$ [22]. Geometry optimization was conducted via the Perdew-Burke-Ernzerhof (PBE) exchange-correlation function within the generalized gradient approximation (GGA) [23]. The convergence tolerance for the total energy and maximum ionic displacement was set as $1.0 \times 10^{-5} \mathrm{eV}$

and $1.0 \times 10^{-3} \AA$, respectively. After completing the geometry optimization, a OTFG ultrasoft potential and generalized gradient approximation (GGA) functional was used to calculate the band structure of $\alpha-\mathrm{AgVO}_{3}$. To further improve the accuracy of band gap, a hybrid HSE03 exchange-correlation functional was employed which has been proven in obtaining a reliable bandgap for complex oxides [24].

\section{Results}

\subsection{Morphology of $\alpha-\mathrm{AgVO}_{3}$ and $\mathrm{GO} / \alpha-\mathrm{AgVO}_{3}$}

Fig. 1 shows the morphologies of the $\alpha-\mathrm{AgVO}_{3}$ and $\mathrm{GO} / \alpha-\mathrm{AgVO} \mathrm{V}_{3}$ with various GO weight percentages. As shown in Fig. 1(a), the pure $\alpha-\mathrm{AgVO}_{3}$ sample shows a flower-like morphology, which is composed of plentiful one-dimensional (1D) $\alpha-\mathrm{AgVO}_{3}$ nanorods with a length of $10-15 \mu \mathrm{m}$ and a width of $100-300 \mathrm{~nm}$. Fig. 1(b-d) 
shows the morphologies of $0.5 \mathrm{GO} / \alpha-\mathrm{AgVO}_{3}, \quad 1.0 \mathrm{GO} / \alpha-\mathrm{AgVO}_{3}$ and 2.0 $\mathrm{GO} / \alpha-\mathrm{AgVO}_{3}$ respectively. The $\alpha-\mathrm{AgVO}_{3}$ nanorods are covered by $2 \mathrm{D}$ GO nanosheets, indicating the formation of heterojunctions. Notably, with the increase of GO content, the accumulation of a large amount of lamellar corrugated GO nanosheets can be observed. The composition of $\mathrm{GO} / \alpha-\mathrm{AgVO}_{3}$ photocatalyst was further characterized by EDS elemental mapping. As shown in Fig. 2, silver, oxygen, and vanadium elements are uniformly distributed on the nanorods, and the corrugated nanosheets correspond to the carbon element, indicating the close coupling of $\alpha-\mathrm{AgVO}_{3}$ nanorods and GO nanosheets.

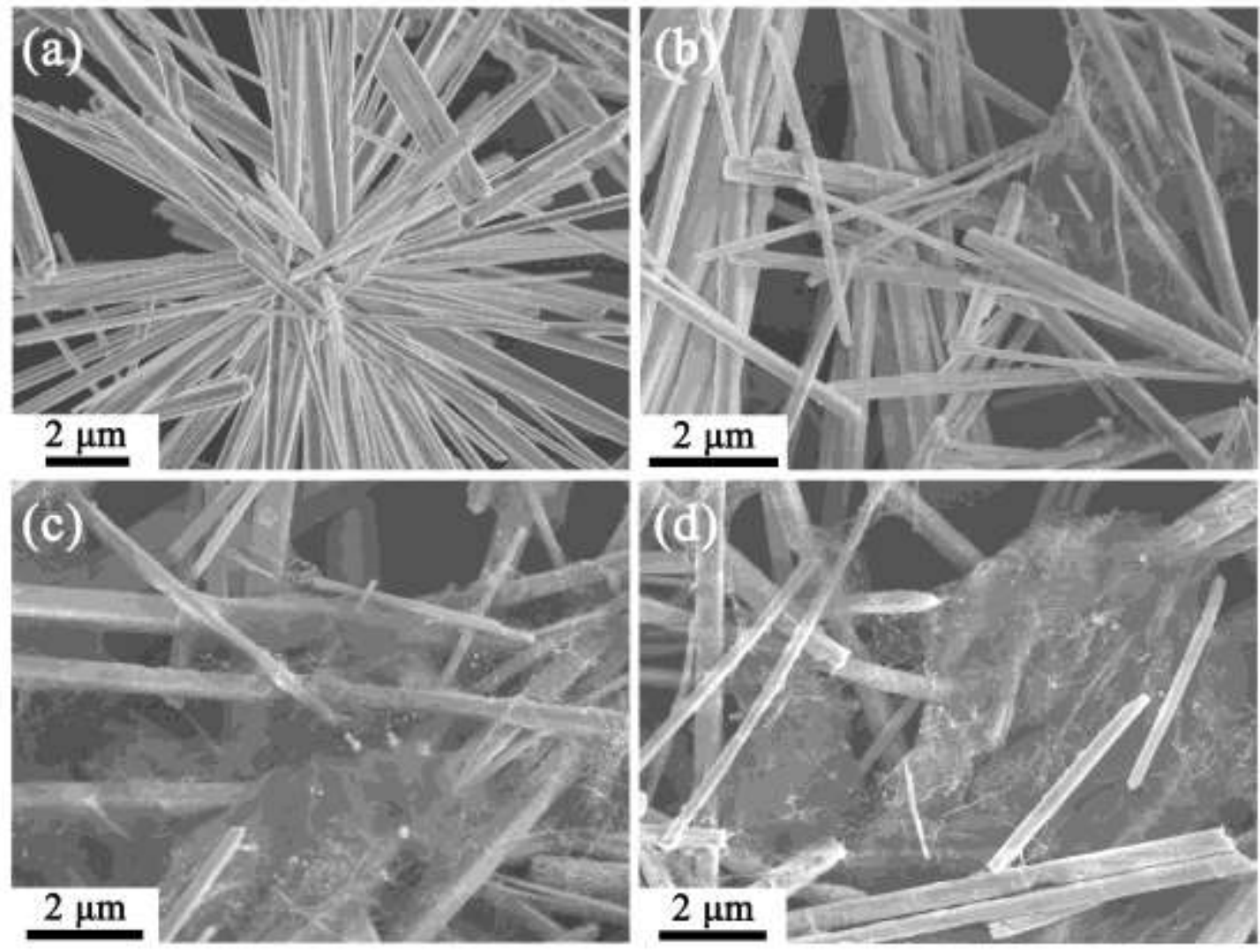

Fig. 1. SEM images of the as-prepared (a) $\alpha-\mathrm{AgVO}_{3}$, (b) $0.5 \mathrm{GO} / \alpha-\mathrm{AgVO}_{3}$, (c) 1.0 $\mathrm{GO} / \alpha-\mathrm{AgVO}_{3}$, (d) $2.0 \mathrm{GO} / \alpha-\mathrm{AgVO}_{3}$. 


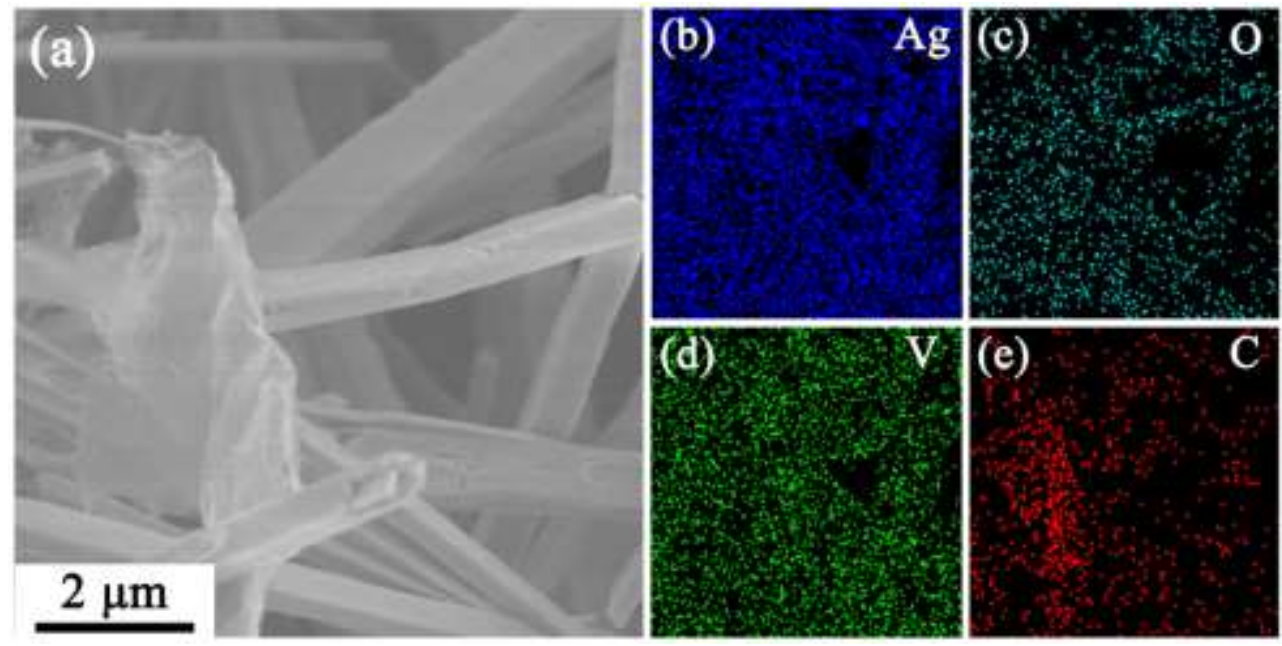

Fig. 2. The SEM image of $0.5 \mathrm{GO} / \alpha-\mathrm{AgVO}_{3}$ (a) and the corresponding EDS

elemental mapping of (b) $\mathrm{Ag}$, (c) $\mathrm{O}$, (d) V, and (e) C.

\subsection{Crystal structure and XRD analysis}

The phase composition and preferred grow direction of $\alpha-\mathrm{AgVO}_{3}$ nanorods were further studied by experimental and simulated XRD patterns. The XRD patterns of the as-prepared $\alpha-\mathrm{AgVO}_{3}$ and $\mathrm{GO} / \alpha-\mathrm{AgVO}_{3}$ are shown in Fig. 3. The $\alpha-\mathrm{AgVO}_{3}$ is well crystallized in a monoclinic structure and no impurity phase can be detected. The main peaks at $2 \theta$ degrees of $12.40^{\circ}, 17.22^{\circ}, 24.95^{\circ}, 27.50^{\circ}, 28.23^{\circ}, 31.51^{\circ}$, $32.12^{\circ}$, and $32.82^{\circ}$ can be ascribed to (110), (200), (220), (310), ( $\left.\overline{2} 21\right),(221),(\overline{131})$ and (002) diffraction planes of $\alpha-\mathrm{AgVO}_{3}$ (according to JCPDS card \#89-4396), respectively. After the introduction of GO, the $2 \theta$ angles of each diffraction peak remains unchanged, indicating that the addition of GO does not affect the phase stability and crystal structure of $\alpha-\mathrm{AgVO}_{3}$. Due to the low percentage and weak reflection of GO, no obvious diffraction peak for GO can be observed in the XRD pattern of $\mathrm{GO} / \alpha-\mathrm{AgVO}_{3}$. 


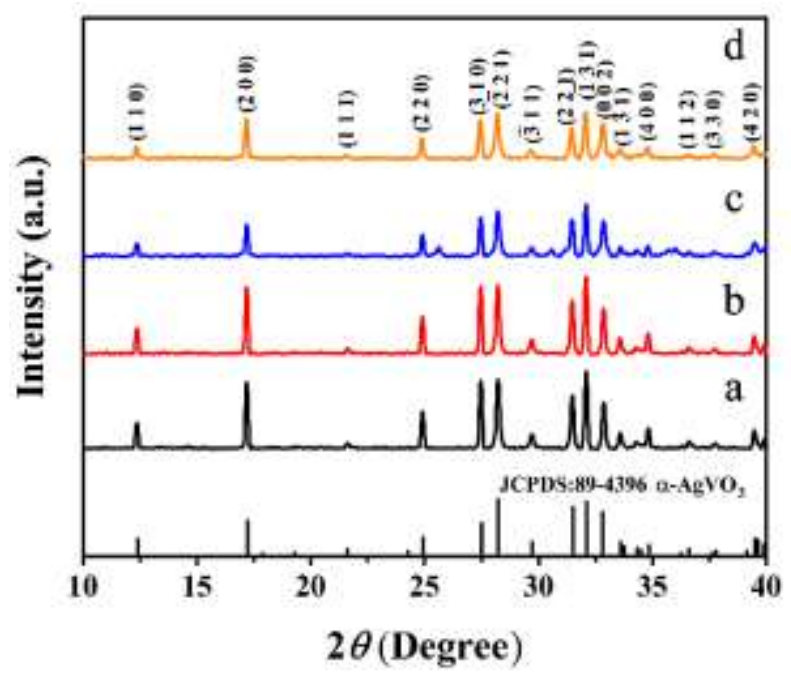

Fig. 3. XRD patterns of (a) $\alpha-\mathrm{AgVO}_{3}$, (b) $0.5 \mathrm{GO} / \alpha-\mathrm{AgVO}_{3}$, (c) $1.0 \mathrm{GO} / \alpha-\mathrm{AgVO}_{3}$ and (d) $2.0 \mathrm{GO} / \alpha-\mathrm{AgVO}_{3}$.

To reveal the growth orientation of $\alpha-\mathrm{AgVO}_{3}$ nanorods, its crystal structure was constructed and optimized (Fig.4 and Table.1), and the comparison of the simulated and experimental XRD patterns of $\alpha-\mathrm{AgVO}_{3}$ is shown in Fig. 5. Fig.4 shows that two $\mathrm{VO}_{4}$ tetrahedrons are connected by sharing vertex and then precipitate with $\mathrm{Ag}$ to crystallize in a monoclinic $\alpha$ phase $\mathrm{AgVO}_{3}$ with space group $C 2 / c$. The geometry optimized lattice constants, atomic positions and bond lengths of $\alpha-\mathrm{AgVO}_{3}$ are shown in Table.1.

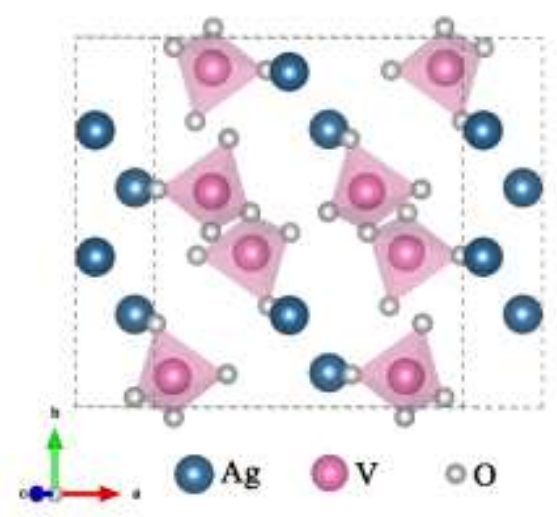

Fig. 4. Crystal structure of $\alpha-\mathrm{AgVO}_{3}$. 
Table 1. Geometry optimized lattice constants, atomic positions and bond lengths of $\alpha-\mathrm{AgVO}_{3}$.

\begin{tabular}{|c|c|c|c|}
\hline Parameter & \multicolumn{3}{|c|}{$\alpha-\mathrm{AgVO}_{3}$} \\
\hline Space & \multicolumn{3}{|c|}{ C 2/c (No.15) } \\
\hline $\begin{array}{l}\text { Lattice } \\
\text { constants }\end{array}$ & \multicolumn{3}{|c|}{$\begin{array}{l}\mathrm{a}=11.2975 \AA \\
\mathrm{b}=10.7962 \AA \\
\mathrm{c}=5.78487 \AA \\
\mathrm{V}=646.534 \AA^{3}\end{array}$} \\
\hline $\begin{array}{l}\text { Atomic } \\
\text { positions }\end{array}$ & \multicolumn{3}{|c|}{$\begin{array}{ll}\text { V1 } & (0.30349,0.41734,0.26496) \\
\text { Ag1 } & (0,0.25195,0.25) \\
\text { Ag2 } & (0,0.59393,0.25) \\
\text { O1 } & (0.13789,0.41143,0.14139) \\
\text { O2 } & (0.14649,0.027,-0.02306) \\
\text { O3 } & (0.36723,0.27405,0.36506)\end{array}$} \\
\hline $\begin{array}{l}\text { Bond } \\
\text { lengths }\end{array}$ & $\begin{array}{l}\text { Bond } \\
\text { O-V } \\
\text { O-V } \\
\text { O-Ag } \\
\text { O-Ag } \\
\text { O-O }\end{array}$ & $\begin{array}{l}\text { Population } \\
0.73 \\
0.73 \\
0.22 \\
0.24 \\
0.00\end{array}$ & $\begin{array}{l}\text { Length }(\AA) \\
1.70684 \\
1.71587 \\
2.17526 \\
2.14763 \\
2.81117 \\
\end{array}$ \\
\hline
\end{tabular}

Based on this geometry optimized crystal structure, the XRD pattern simulation was carried out through the Reflex code in Materials Studio software (Accelrys Software Inc., San Diego, CA, USA) using CuKa radiation of $\lambda=1.5406 \AA$, step size of $0.02^{\circ}$. The preferred $c$-axis orientation was imposed by the Rietveld-Toraya method and the simulated morphology was calculated based on the theory of Donnay-Harker [25-27]. According to Scherrer's formula, the grain size difference in different directions will result in different broadening degree of diffraction peaks. Thus, as shown in Fig. 5, the simulated XRD pattern of $\alpha-\mathrm{AgVO}_{3}$ nanorods with a growth direction that perpendicular to the (002) crystal plane is closer to the experimental result, compared with that of the power reflection without orientation. Meanwhile, the simulated morphology shown in Fig.5(d) further confirms that the $\alpha-\mathrm{AgVO}_{3}$ nanorods prefers to growth along $c$-axis that perpendicular to (002) crystal 
plane.

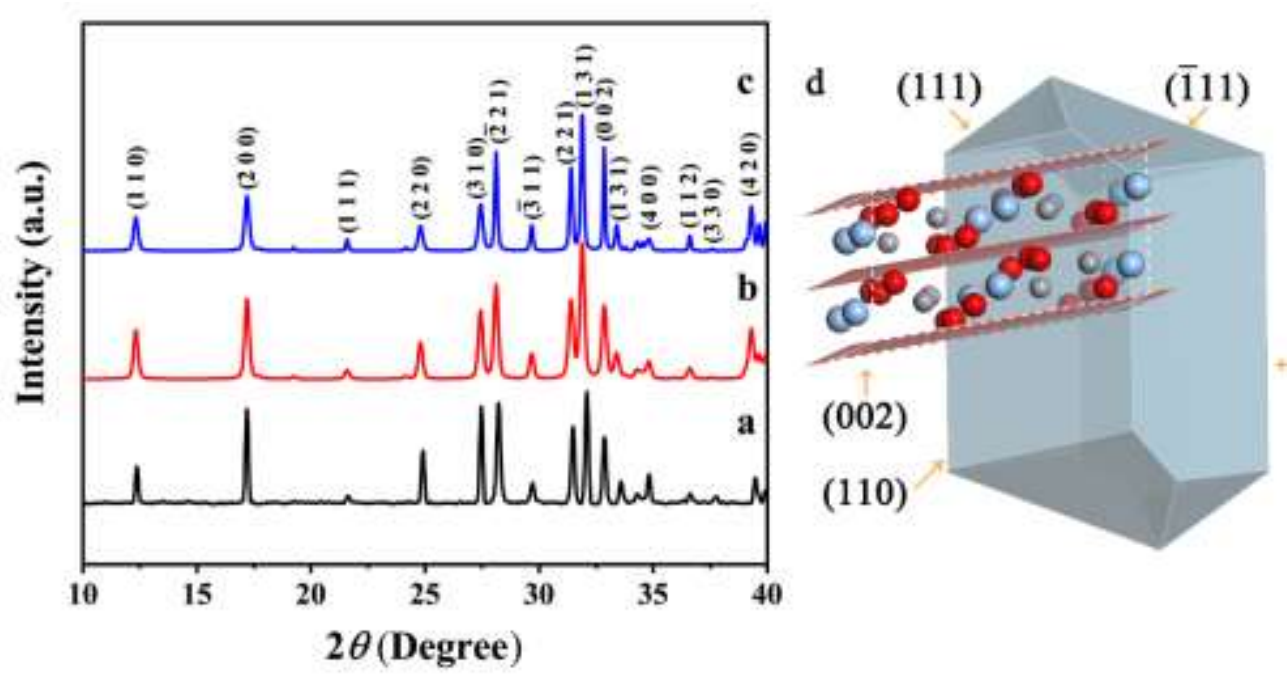

Fig. 5. Experimental XRD patterns of $\alpha-\mathrm{AgVO}_{3}$ (a), together with simulated XRD patterns of powder (b) and $c$-axis oriented particle (c). (d) the calculated morphology based on the theory of Donnay-Harker. (The purple crystal is the $\alpha-\mathrm{AgVO}_{3}$ nanorod, and the plane is the $\left(\begin{array}{lll}0 & 0 & 2\end{array}\right)$ plane. $)$

\subsection{FTIR and Raman characterization}

In order confirm the presence of $\mathrm{GO}$ in the $\mathrm{GO} / \alpha-\mathrm{AgVO}_{3}$ photocatalysts, Fourier transform infrared spectroscopy (FTIR Spectroscopy) and Raman spectroscopy were employed. As shown in the FTIR spectra (Fig. 6a), the bands related to the $\mathrm{VO}_{4}$ tetrahedron, including $v(\mathrm{~V}-\mathrm{O}-\mathrm{V})\left(515 \mathrm{~cm}^{-1}\right), \delta(\mathrm{V}-\mathrm{O})\left(636 \mathrm{~cm}^{-1}\right)$, and $v(\mathrm{~V}=\mathrm{O})(660$, 895,929 , and $964 \mathrm{~cm}^{-1}$ ) vibrational modes are observed, as well as bands at $846 \mathrm{~cm}^{-1}$ for $v(\mathrm{Ag}-\mathrm{O}-\mathrm{V})$ stretching modes $[28,29]$. For $\mathrm{GO} / \alpha-\mathrm{AgVO}_{3}$, in addition to the bands mentioned above, typical peaks of $\mathrm{C}-\mathrm{O}$ stretching and $\mathrm{C}-\mathrm{C} s p^{2}$ in-plane vibration emerge at $1057 \mathrm{~cm}^{-1}$ and $1624 \mathrm{~cm}^{-1}$, demonstrating the successfully introduction of GO [30]. In fact, GO is more sensitive to Raman spectroscopy. As shown in Fig. 6b, two typical GO peaks at about 1340 and $1597 \mathrm{~cm}^{-1}$ appear in the spectra of 
$\mathrm{GO} / \alpha-\mathrm{AgVO}_{3}$, which are attributed to the disordered $s p^{2}$ carbon (D-band) and well-ordered graphite (G-band) respectively [31, 32]. With the increase of GO contents, the intensity of $\mathrm{D}$ and $\mathrm{G}$ peaks also increase. Moreover, the main characteristic vibration peaks of $\alpha-\mathrm{AgVO}_{3}$ are all observed as well. In Raman spectra of all the samples, the strongest band at $919 \mathrm{~cm}^{-1}$ may originate from symmetric stretching of $\mathrm{V}-\mathrm{O}-\mathrm{Ag}$ or $\mathrm{O}-\mathrm{V}-\mathrm{O}$ vibrations. The band at $897 \mathrm{~cm}^{-1}$ is linked with the stretching vibrations of $\mathrm{Ag}-\mathrm{O}-\mathrm{Ag}$, while the band at $875 \mathrm{~cm}^{-1}$ represents the stretching vibrations of the $\mathrm{V}-\mathrm{O}-\mathrm{Ag}$. The bands appearing at 850, 762, 627, and 528 $\mathrm{cm}^{-1}$ are related to the asymmetric and symmetric stretching modes of $\mathrm{V}-\mathrm{O}-\mathrm{V}$ [33-35]. Based on the FTIR and Raman spectra results, the composition of $\mathrm{GO} / \alpha-\mathrm{AgVO}_{3}$ are further confirmed. All the foregoing results demonstrate that a serial of $\alpha-\mathrm{AgVO}_{3}(002) / / \mathrm{GO}(002)$ heterostructure photocatalysts have been successfully prepared.
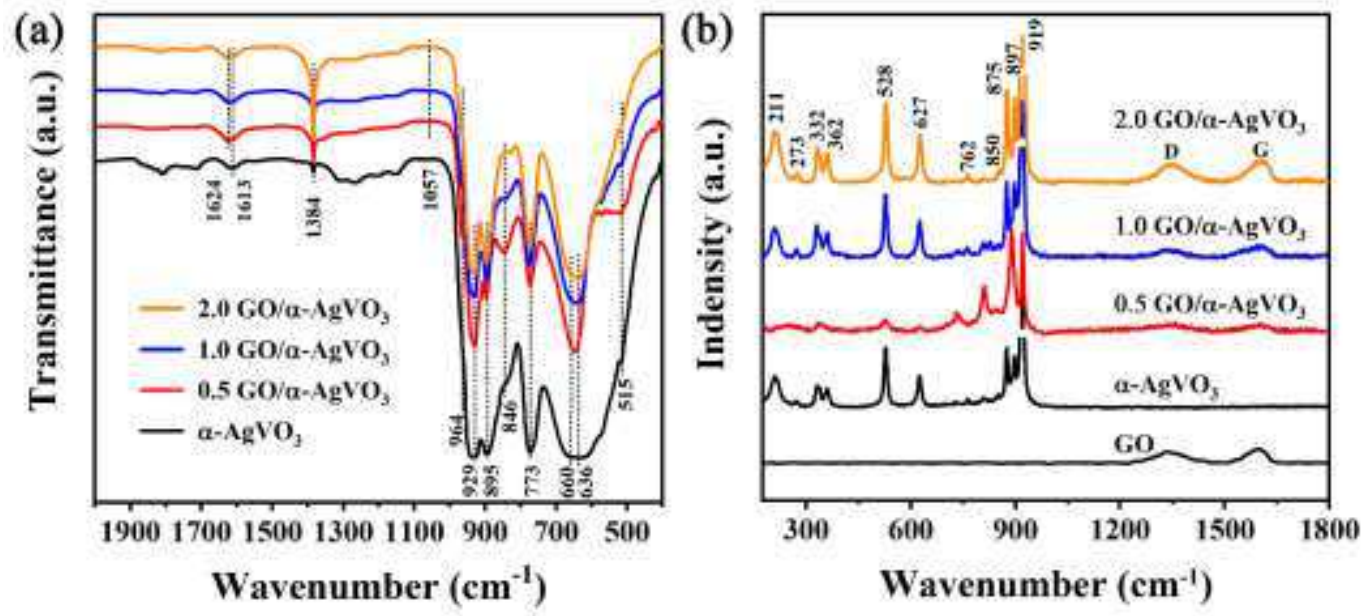

Fig. 6. (a) FTIR spectra and (b) Raman spectra of $\alpha-\mathrm{AgVO}_{3}$ and $\mathrm{GO} / \alpha-\mathrm{AgVO}_{3}$ with different GO contents (in 0.5 wt. $\%, 1.0$ wt. $\%$, and 2.0 wt. $\%$ ).

\subsection{Photocatalytic performance}


The photocatalytic activities of $\alpha-\mathrm{AgVO}_{3}$ and $\mathrm{GO} / \alpha-\mathrm{AgVO}_{3}$ are evaluated in terms of the photodegradation of RhB. Fig. 7(a) shows the variation of $\mathrm{RhB}$ concentration with irradiation time of visible light. It is obvious that the introduction of GO significantly improves the photocatalytic performance compared with pure $\alpha-\mathrm{AgVO}_{3}$. After $40 \mathrm{~min}$ of irradiation, the $0.5 \mathrm{GO} / \alpha-\mathrm{AgVO}_{3}$ photocatalyst displays the highest photocatalytic activity with $90.0 \% \mathrm{RhB}$ degradation, while few $\mathrm{RhB}$ is removed by pure $\alpha-\mathrm{AgVO}_{3}$. Quantitatively, the photocatalytic degradation of $\mathrm{RhB}$ can be described by pseudo-first-order dynamic process and its kinetics can be expressed as:

$$
\ln \left(\frac{C_{0}}{C}\right)=k_{1} t
$$

where $C$ is the concentration of the $\mathrm{RhB}$ at time $t, C_{0}$ is the initial concentration of the $\mathrm{RhB}$ solution, and the slope $k_{1}$ is the apparent reaction rate constant. As shown in Fig. 7(b), the $0.5 \mathrm{GO} / \alpha-\mathrm{AgVO}_{3}$ photocatalyst has the maximum rate constant of 0.0584 $\min ^{-1}$ which is 18 times higher than that of pure $\alpha-\mathrm{AgVO}_{3}$, revealing the superior photocatalytic activity of the $\mathrm{GO} / \alpha-\mathrm{AgVO}_{3}$. With the increase of $\mathrm{GO}$ contents, the degradation efficiency decreases probably due to the aggregation of GO nanosheets as shown in SEM images in Fig. 1. Compared with the performance of other SVOs and traditional $\mathrm{TiO}_{2}$ photocatalysts under the similar experimental conditions [16,36-42], the $\mathrm{GO} / \alpha-\mathrm{AgVO}_{3}$ photocatalyst shows outstanding performance with fast rate and large degradation ratio as shown in Table 2. 

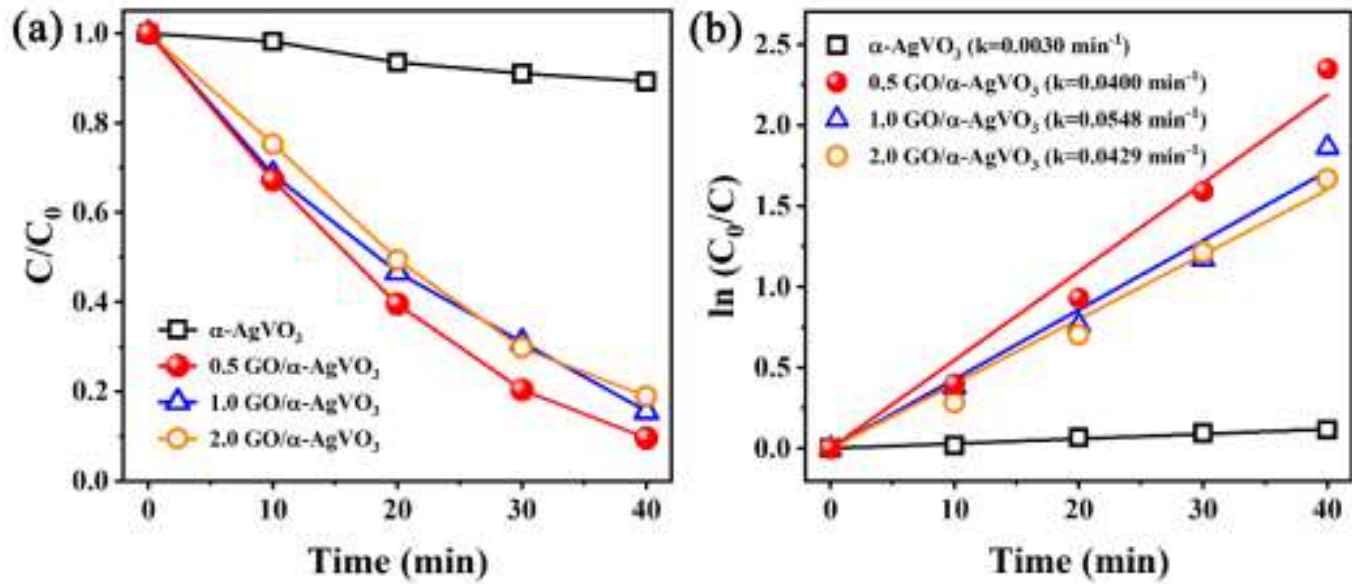

Fig. 7. Photocatalytic degradation curves of $\mathrm{RhB}$ (a) and the corresponding

pseudo-first-order kinetic curves (b).

Table 2. RhB photodegradation properties of recently reported SVOs and other photocatalysts.

\begin{tabular}{ccccc}
\hline Photocatalyst & $\begin{array}{c}\text { Loading } \\
\text { (Photocatalyst/Dye) }\end{array}$ & $\begin{array}{c}\text { Degradation } \\
\text { ratio }\end{array}$ & $\begin{array}{c}\text { Apparent rate } \\
\text { constant } k\left(\mathrm{~min}^{-1}\right)\end{array}$ & Reference \\
\hline $\mathrm{TiO}_{2}$ & $100 \mathrm{mg} / 1 \mathrm{mg}$ & $12 \%$ & 0.0060 & Wang, et al [36] \\
$\alpha-\mathrm{AgVO}_{3} / \alpha-\mathrm{FeOOH}$ & $50 \mathrm{mg} / 0.24 \mathrm{mg}$ & $45 \%$ & 0.0194 & Sun, et al [16] \\
$\beta-\mathrm{AgVO}_{3} / \mathrm{Ag}_{3} \mathrm{VO}_{4}$ & $50 \mathrm{mg} / 0.3 \mathrm{mg}$ & $76 \%$ & 0.0340 & Gao, et al [37] \\
$\beta-\mathrm{AgVO}_{3} / \mathrm{BiVO}_{4}$ & $50 \mathrm{mg} / 1 \mathrm{mg}$ & $69 \%$ & 0.0206 & Wang, et al [38] \\
$\beta-\mathrm{AgVO}_{3} / \mathrm{AgBr}$ & $30 \mathrm{mg} / 0.5 \mathrm{mg}$ & $23 \%$ & 0.0183 & Zhang, et al [39] \\
$\mathrm{Ag}_{4} \mathrm{~V}_{2} \mathrm{O}_{7}$ & $80 \mathrm{mg} / 0.38 \mathrm{mg}$ & $38 \%$ & - & Wang, et al [40] \\
$\mathrm{g}_{-} \mathrm{C}_{3} \mathrm{~N}_{4} / \mathrm{Ag}_{4} \mathrm{~V}_{2} \mathrm{O}_{7}$ & $100 \mathrm{mg} / 1 \mathrm{mg}$ & $55 \%$ & 0.015 & Zhang, et al [41] \\
$\mathrm{Ag}_{3} \mathrm{VO}_{4}$ & $2 \mathrm{~g} / 4.8 \mathrm{mg}$ & $32 \%$ & 0.0155 & Zhang, et al [42] \\
$\mathrm{GO} / \alpha-\mathrm{AgVO}_{3}$ & $15 \mathrm{mg} / 0.15 \mathrm{mg}$ & $90 \%$ & 0.0584 & This work \\
\hline
\end{tabular}

a The degradation percentage is collected at 40 min visible-light irradiation for better comparison.

\section{Discussion}

\subsection{Electronic structure and bandgaps}

To shed light on the photocatalytic mechanism, it is necessary to analyze the electronic structure of $\alpha-\mathrm{AgVO}_{3}$. Fig. 8 shows the band structure of $\alpha-\mathrm{AgVO}_{3}$ along the high symmetry line in the Brillouin zone calculated by GGA (Fig.8a) and HSE03 functional (Fig.8b), respectively. The $\alpha-\mathrm{AgVO}_{3}$ is a direct band gap semiconductor 
because the top of valence bands and bottom of conduction bands are at the Gamma point simultaneously. The direct band gap of $\alpha-\mathrm{AgVO}_{3}$ obtained from GGA functional is $1.9 \mathrm{eV}$ because the GGA functional generally underestimated the experimental band gaps. To improve the accuracy of band gap calculation, a hybrid functional [43] based on a screened Coulomb potential HSE03 was used in DFT calculation. As shown in Fig. 8(b), the band gap obtained by HSE03 functional is $3.0 \mathrm{eV}$ which is believed more reliable and closer to the experimental values for complex oxides [24,44].
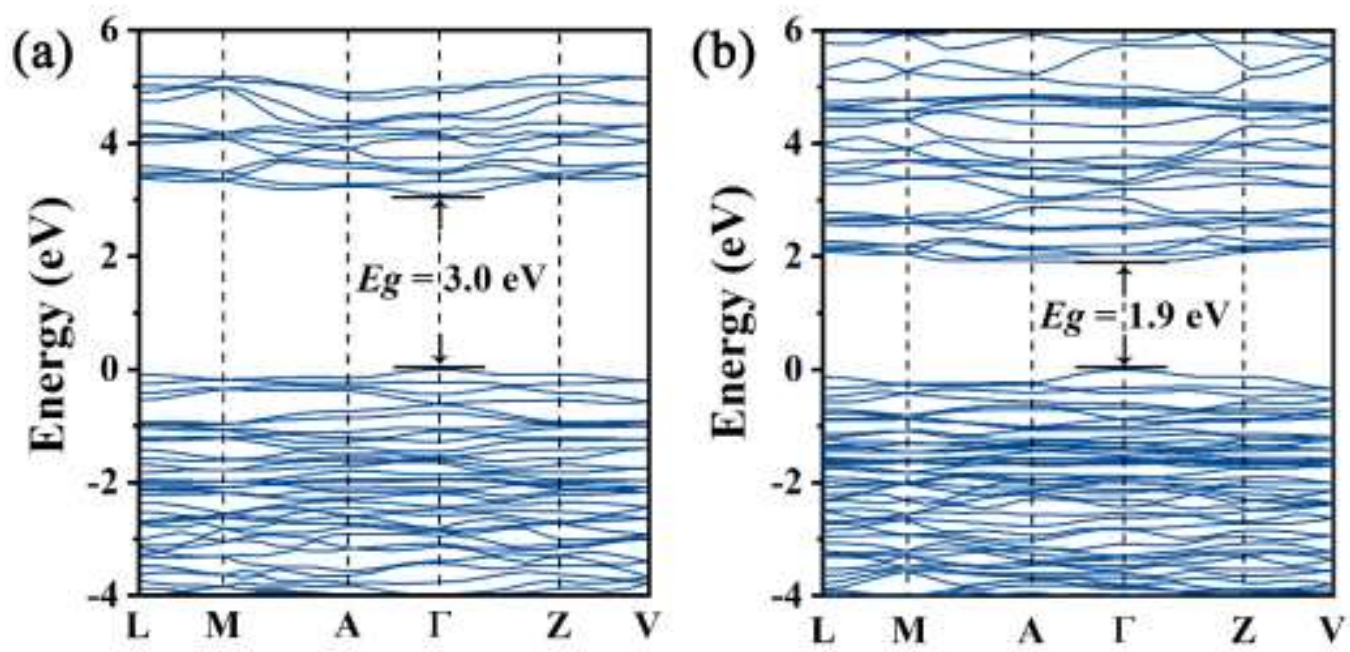

Fig. 8. Band structure of $\alpha-\mathrm{AgVO}_{3}$ along the high symmetry line in the Brillouin zone calculated by (a) GGA and (b) HSE03 functional.

To analyze the optical properties and obtain the experimental bandgaps, UV-vis absorption spectra of $\alpha-\mathrm{AgVO}_{3}$ and $\mathrm{GO} / \alpha-\mathrm{AgVO}_{3}$ nanorods are collected. As shown in Fig. 9(a), the absorbance edge of $\alpha-\mathrm{AgVO}_{3}$ is around $540 \mathrm{~nm}$. After the introduction of 0.5 and 1.0 wt.\% GO, the absorption band edge shows an obvious red-shift to the visible-light region, indicating that a small amount of GO is helpful for visible light absorption. However, 2.0 wt.\% GO addition causes the absorption band edge to shift in the opposite direction, i.e., visible-light absorption and performance 
are weakened, which is consistent with the photocatalysis activities.

The band gap of $\alpha-\mathrm{AgVO}_{3}$ can be obtained by the Kubelka-Munk method [45].

$$
(\alpha h v)^{n}=A\left(h v-E_{g}\right)
$$

where $\alpha, h, v, A$, and $E_{g}$ are the absorption coefficient, Planck's constant, light frequency, proportionality constant, and optical bandgap, respectively. The index $n$ depends on the electronic transition of the crystalline semiconductor, $n=0.5$ for indirect-gap semiconductor and $n=2$ for direct-gap semiconductor. For $\alpha-\mathrm{AgVO}_{3}$ crystal, $\mathrm{n}$ equals 2 , because $\alpha-\mathrm{AgVO}_{3}$ is a direct transition type of semiconductors, according to the band structure in Fig. 8. The Kubelka-Munk plots are shown in Fig. 9(b). The band gap energy can be estimated from the intercept of the tangent to the plot of $(\alpha h v)^{2}$ vs energy $(h v)$. The band gap of $\alpha-\mathrm{AgVO}_{3}$ is $2.56 \mathrm{eV}$, which is approximate to the calculated bandgap from HSE03. The bandgaps for $0.5,1.0$ and $2.0 \mathrm{GO} / \alpha-\mathrm{AgVO}_{3}$ are $2.59,2.62$, and $2.26 \mathrm{eV}$, i.e., the addition of 0.5 and $1.0 \mathrm{wt} . \%$ GO can help to increase the band gap, which is beneficial for the separation of photogenerated carriers. However, continues to increase the GO content will narrow the band gap due to the conductive nature of GO, resulting in the decrease of photocatalytic performance. Therefore, the theoretical and experimental analysis of band structure demonstrates that $\alpha-\mathrm{AgVO}_{3}$ is a visible-light-responsible photocatalyst, and the combination with an appropriate amount of GO helps to expand the absorption of visible light and improve the photocatalytic efficiency. 

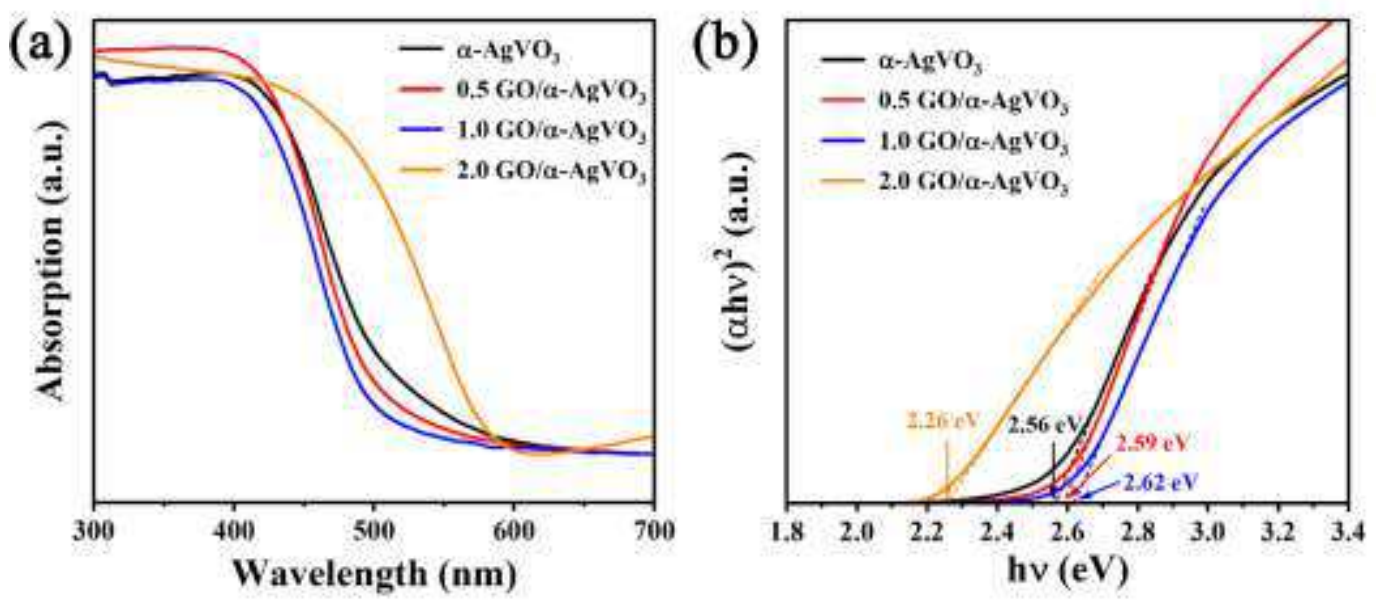

Fig. 9. UV-vis diffuse reflectance spectra (a) and Kubelka-Munk plot (b) of $\alpha-\mathrm{AgVO}_{3}$ and $\mathrm{GO} / \alpha-\mathrm{AgVO}_{3}$ with different $\mathrm{GO}$ contents (in 0.5 wt. $\%, 1.0$ wt. $\%$, and 2.0 wt. $\%)$.

To further analyze the band structure, ultraviolet photoelectron spectroscopy (UPS) is implemented to ascertain the valence band energy $\left(E_{v}\right)$, as shown in Fig. 10(a). The $E_{v}$ of $\alpha-\mathrm{AgVO}_{3}$ is calculated to be $6.61 \mathrm{eV}$ via subtracting the width of the UPS spectra from the excitation energy of $21.22 \mathrm{eV}$ [46]. The conduction band energy $\left(E_{c}\right)$ is thus estimated at $4.05 \mathrm{eV}$ from the equation: $E_{C}=E_{V}-E_{g}$. Considering the reference standard for which $0 \mathrm{eV}$ vs. NHE (Normal hydrogen electrode) equals -4.44 $\mathrm{eV}$ vs. $E_{a v s}$ (Vacuum level), the $E_{V}$ and $E_{C}$ of $\alpha-\mathrm{AgVO}_{3}$ are converted to -0.39 and 2.27 $\mathrm{eV}$ (vs. NHE) [47]. The energy position of $\alpha-\mathrm{AgVO}_{3}$ is illustrated in Fig. 10(b) [48], which provides clear guidance for the subsequent proposal of the photocatalytic mechanism. 

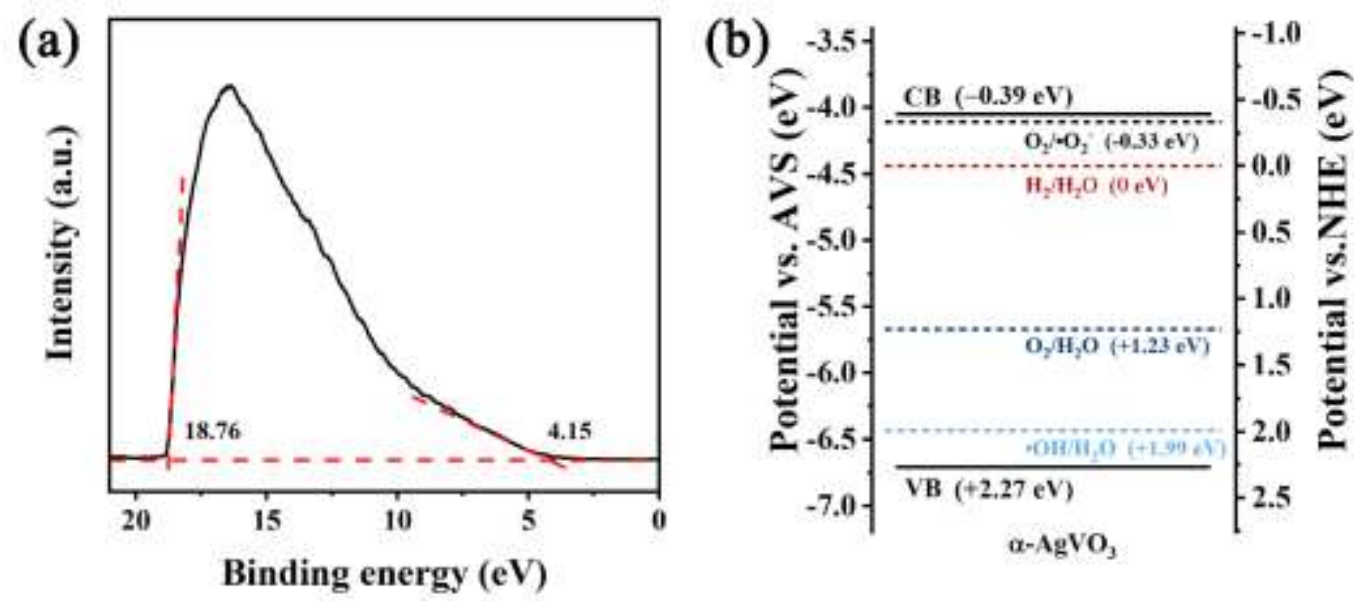

Fig. 10. UPS spectra (a) and the band structure diagram of $\alpha-\mathrm{AgVO}_{3}(\mathrm{~b})$.

\subsection{The role of $\mathrm{GO} / \alpha-\mathrm{AgVO}_{3}$ heterojunction}

According to the structure and morphology characterization and simulated morphology in section 3, the $\alpha-\mathrm{AgVO}_{3}$ (002) // GO (002) heterojunction is successfully constructed in the $\mathrm{GO} / \alpha-\mathrm{AgVO}_{3}$ photocatalyst. The surface chemical composition and the interaction of $\mathrm{GO}$ and $\alpha-\mathrm{AgVO}_{3}$ were further confirmed by XPS. The full range XPS spectrum in Fig. 11(a) confirms the presence of C, Ag, V, and O elements in the $0.5 \mathrm{GO} / \alpha-\mathrm{AgVO}_{3}$ without impurities. The high-resolution XPS spectra of $\mathrm{Ag} 3 d$, V $2 p$, and $\mathrm{O} 1 s$ are shown in Fig. 11(b-d). In Fig. 11(b), the peaks that emerged at the binding energy of $367.5 \mathrm{eV}$ and $373.5 \mathrm{eV}$ correspond to $\mathrm{Ag} 3 d_{5 / 2}$ and $\operatorname{Ag} 3 d_{3 / 2}$ orbits. The XPS spectra of $\mathrm{V} 2 p$ show two specific peaks of pentavalent vanadium at the binding energy of $516.4 \mathrm{eV}$ and $523.8 \mathrm{eV}$ [49], as shown in Fig.11(c). The O $1 s$ spectra shown in Fig.11(d) can be split into three peaks at $529.9 \mathrm{eV}, 530.4$ $\mathrm{eV}$, and $532.1 \mathrm{eV}$, which are attributed to the binding energies of lattice oxygen, oxygen defects and adsorbed oxygen species [50]. After the introduction of GO, a new $\mathrm{C}=\mathrm{O}$ peak appears at $533.4 \mathrm{eV}$ in the $\mathrm{O} 1 \mathrm{~s}$ spectrum [51]. More importantly, 
peaks of $\mathrm{Ag} 3 d, \mathrm{~V} 2 p$, and $\mathrm{O} 1 s$ in $0.5,1.0$, and $2.0 \mathrm{GO} / \alpha-\mathrm{AgVO}_{3}$ are all shifted towards the high binding energy, indicating the electron migration from $\alpha-\mathrm{AgVO}_{3}$ to GO nanosheet happens, which can improve the separation efficiency of photogenerated carriers and enhance photocatalytic performance [12].

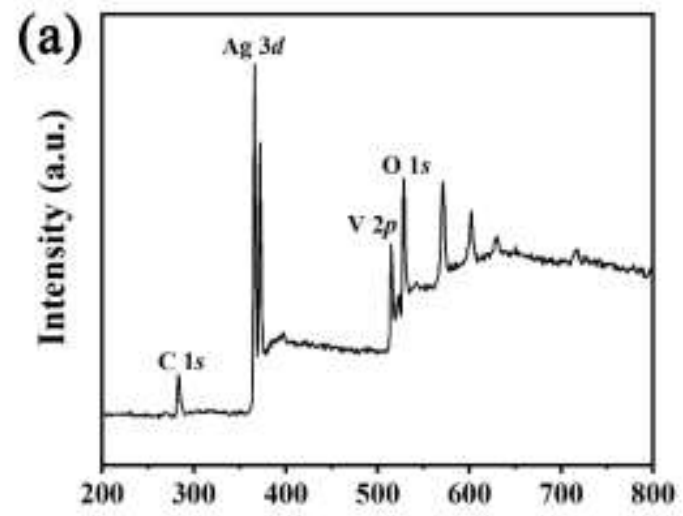

Binding energy (eV)

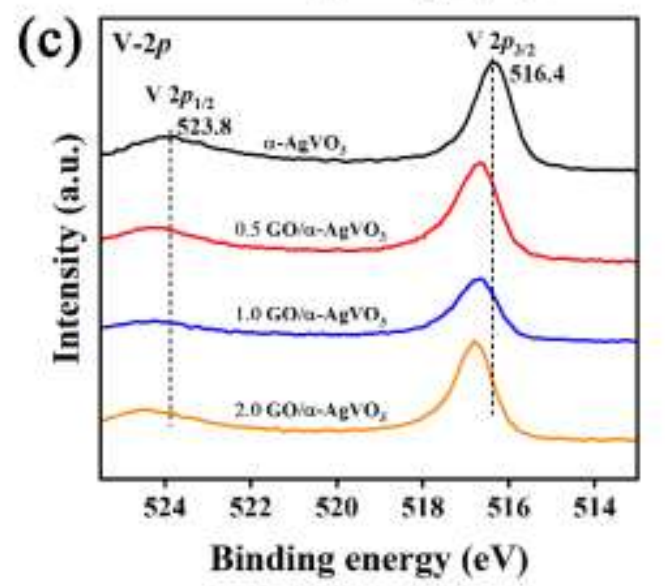

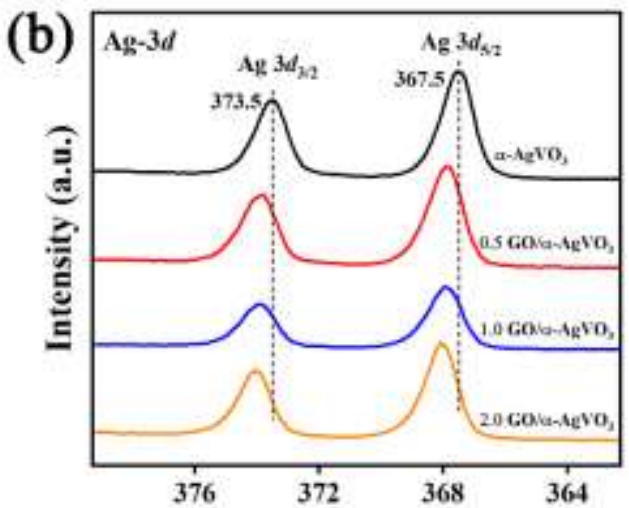

Binding energy (eV)

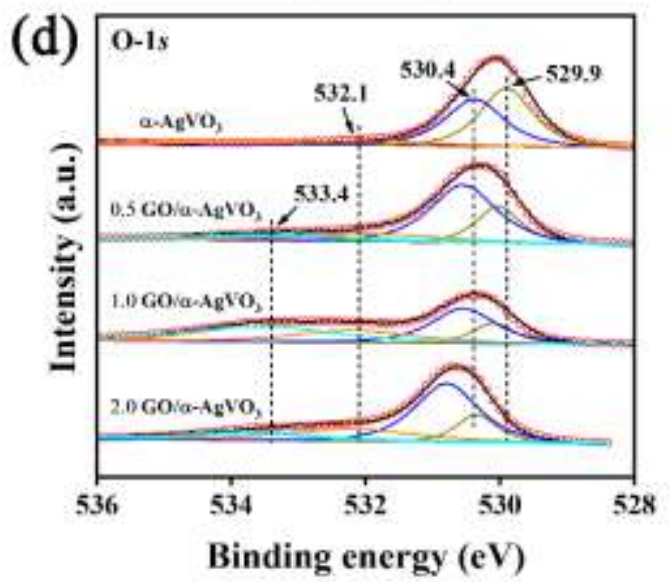

Fig 11. Full-scale XPS survey spectrum for $0.5 \mathrm{GO} / \alpha-\mathrm{AgVO}_{3}$ (a) and the XPS spectra of (b) $\mathrm{Ag} 3 d$, (c) $\mathrm{V} 2 p$ and $\mathrm{O} 1 s$ for $\mathrm{GO} / \alpha-\mathrm{AgVO}_{3}$ with different $\mathrm{GO}$ contents (in 0.5 wt. $\%, 1.0$ wt. $\%$, and 2.0 wt. $\%)$.

\subsection{Mechanism for the enhanced photocatalytic performance of the $\mathrm{GO} / \mathrm{\alpha}-\mathrm{AgVO}$}

Based on the foregoing analysis, the possible mechanism of the $\mathrm{GO} / \alpha-\mathrm{AgVO}_{3}$ photocatalyst is proposed and illustrated in Fig. 12. When the visible-light shines on the surface of $\mathrm{GO} / \alpha-\mathrm{AgVO}_{3}$ photocatalyst, the electrons $\left(\mathrm{e}^{-}\right)$at valance band (VB) are 
excited to conduct band (CB), leaving an equal number of holes $\left(\mathrm{h}^{+}\right)$at $\mathrm{VB}$, as presented in equation (3). The electrons in the conduction band of $\alpha-\mathrm{AgVO}_{3}$ can transfer to the surface of $\mathrm{GO}$ through the $\alpha-\mathrm{AgVO}_{3}(002) / / \mathrm{GO}(002)$ heterojunction, promoting the separation efficiency of photogenerated carriers. Taking advantage of the conductivity of GO's $\pi-\pi$ graphitic carbon network, the lifetime of electrons can be further prolonged [52], which is helpful to improve the photocatalytic performance. Then, the $\mathrm{h}^{+}$at VB can produce hydroxyl radicals $(\bullet \mathrm{OH})$ by reacting with $\mathrm{H}_{2} \mathrm{O}$, while the $\mathrm{e}^{-}$at $\mathrm{GO}$ can generate superoxide radicals. These radicals and holes with high oxidative activity can degrade organic pollutants like RhB efficiently by the possible equations as follow:

$\alpha-\mathrm{AgVO}_{3}+h v \rightarrow \alpha-\mathrm{AgVO}_{3}\left(\mathrm{e}^{-}+\mathrm{h}^{+}\right)$

$\mathrm{e}^{-}\left(\alpha-\mathrm{AgVO}_{3}\right)+\mathrm{GO} \rightarrow \alpha-\mathrm{AgVO}_{3}+$ injected $\mathrm{e}^{-}(\mathrm{GO})$

$\alpha-\mathrm{AgVO}_{3}\left(\mathrm{~h}^{+}\right)+\mathrm{H}_{2} \mathrm{O} \rightarrow \cdot \mathrm{OH}+\mathrm{H}^{+}$

$\mathrm{e}^{-}\left(\mathrm{GO} / \alpha-\mathrm{AgVO}_{3}\right)+\mathrm{O}_{2} \rightarrow \cdot \mathrm{O}^{2-}$

$\mathrm{RhB}+\alpha-\mathrm{AgVO}_{3}\left(\mathrm{~h}^{+}\right) / \cdot \mathrm{O}^{2-} / \bullet \mathrm{OH} \rightarrow$ intermediates $\rightarrow \mathrm{CO}_{2}+\mathrm{H}_{2} \mathrm{O}$

Therefore, suitable band structure and band gap for visible light absorption are fundamental factors for visible-light-responsive photocatalyst, and their photocatalytic performances can be further improved by inhibiting the recombination of photogenerated carriers through heterostructure design. 


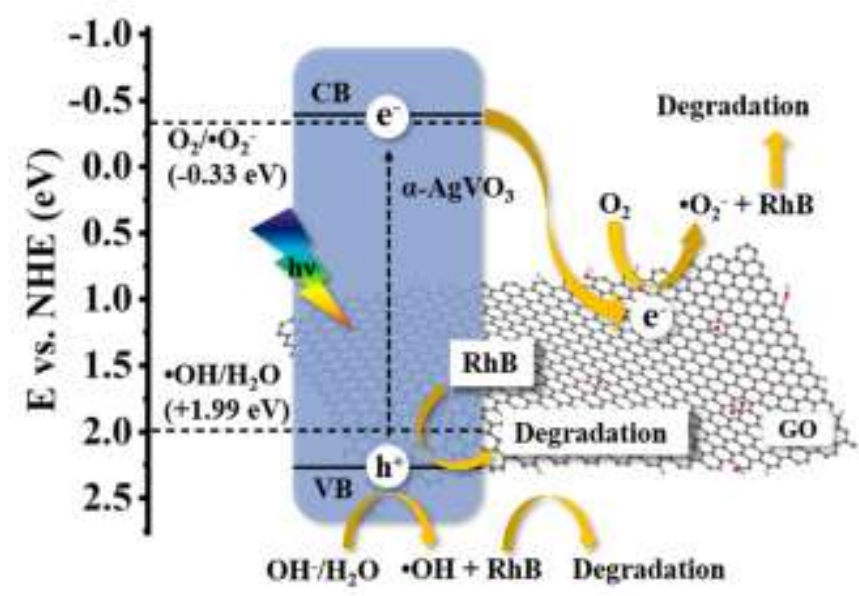

Fig. 12. Proposed photocatalytic mechanism of $\mathrm{GO} / \alpha-\mathrm{AgVO}_{3}$ photocatalyst.

\section{Conclusions}

In summary, novel visible-light-responded photocatalysts including $0.5 \mathrm{wt} \%$ $\mathrm{GO} / \alpha-\mathrm{AgVO}_{3}, 1.0 \mathrm{wt} \% \mathrm{GO} / \alpha-\mathrm{AgVO}_{3}, 2.0 \mathrm{wt} \% \mathrm{GO} / \alpha-\mathrm{AgVO}_{3}$, and pure $\alpha-\mathrm{AgVO}_{3}$ were successfully synthesized by a facile in-situ coprecipitation method, and their structure, morphology, photocatalytic performance and mechanism were revealed. SEM analyses indicate that the as-prepared photocatalysts have a flower-like morphology, wherein the petals consist of $\alpha-\mathrm{AgVO}_{3}$ nanorods with a length of a few microns and then GO nanosheets are uniformly covered on these nanorods. XRD analyses demonstrate that $\alpha-\mathrm{AgVO}_{3}$ is well crystallized in a monoclinic $\alpha$ phase with space group $C 2 / c$. Through the reflex stimulation, it can be confirmed that the $\alpha-\mathrm{AgVO}_{3}$ nanorods prefer to grow along the $c$ axis. Raman, FTIR, and XPS spectra demonstrate the existence of GO nanosheets, and finally, confirm the formation of $\alpha-\mathrm{AgVO}_{3}(002)$ // GO (002) heterojunction. The successful introduction of GO nanosheets and construction of heterostructure might have unique effect on the photocatalytic performance. 
Compared with pure $\alpha-\mathrm{AgVO}_{3}$ and other SVOs, $0.5 \mathrm{GO} / \alpha-\mathrm{AgVO}_{3}$ displays the optimal photodegradation efficiency of $90 \% \mathrm{RhB}$ removal with the maximum rate constant of $0.0584 \mathrm{~min}^{-1}$ which is tens of times higher than that of pure $\alpha-\mathrm{AgVO}_{3}$ and other SVOs. Proper band gap and efficient photocarrier separation play significant roles in high photocatalytic performance of $\mathrm{GO} / \alpha-\mathrm{AgVO}_{3}$. Electronic structure and band gap calculation by HSE03 functional and optical properties analysis reveals that is $\alpha-\mathrm{AgVO}_{3}$ a direct-gap semiconductor with a band gap of about $2.56 \mathrm{eV}$ which is suitable for visible light absorption and irradiation. Then, the $\mathrm{GO}$ and $\alpha-\mathrm{AgVO}_{3}$ heterojunction facilitates the transfer of photogenerated electrons, which overcomes the obstacle of rapid recombination of carriers and further boosts the photocatalytic activity. Moreover, some active species like $\mathrm{h}^{+}, \cdot \mathrm{O}^{2-}$ and $\cdot \mathrm{OH}$ generated by the photochemical reaction can help degrade organic pollutants like RhB. With the advantage of suitable band gap, efficient photocarriers separation and superior photocatalytic performance, $\mathrm{GO} / \alpha-\mathrm{AgVO}_{3}$ is a promising photocatalyst for the visible-light degradation of pollutants and water environmental protection.

\section{Acknowledgement}

The authors gratefully acknowledge the financial support of the key laboratory Foundation of the Science and Technology on Advanced Functional Composite Laboratory (Grant No. 6142906200509), the Natural Science foundation of Jiangsu Province Colleges (Grant No. 20KJB430017) and NUPTSF (Grant No. NY219162). 


\section{References}

1.Low J, Yu J, Jaroniec M, et al. Heterojunction photocatalysts. Adv Mater 2017, 29: 1601694.

2.Ju P, Wang Y, Sun Y, et al. In-situ green topotactic synthesis of a novel Z-scheme $\mathrm{Ag} @ \mathrm{AgVO}_{3} / \mathrm{BiVO}_{4}$ heterojunction with highly enhanced visible-light photocatalytic activity. $J$ Colloid Interf Sci 2020, 579: 431-447.

3.Jeong S Y, Shin HM, Jo YR, et al. Plasmonic silver nanoparticle-impregnated nanocomposite $\mathrm{BiVO}_{4}$ photoanode for plasmon-enhanced photocatalytic water splitting. J Phys Chem C 2018, 122: 7088-7093.

4.Sadeghzadeh-Attar A. Photocatalytic degradation evaluation of $\mathrm{N}-\mathrm{Fe}$ codoped aligned $\mathrm{TiO}_{2}$ nanorods based on the effect of annealing temperature. J Adv Ceram 2020, 9: 107-122.

5.Su T, Shao Q, Qin Z, et al. Role of interfaces in two-dimensional photocatalyst for water splitting. ACS Catal 2018, 8: 2253-2276.

6.Nomikos GN, Panagiotopoulou P, Kondarides DI, et al. Kinetic and mechanistic study of the photocatalytic reforming of methanol over $\mathrm{Pt} / \mathrm{TiO}_{2}$ catalyst. Appl Catal B Environ 2014, 146: 249-257.

7.Zhao W, Guo Y, Wang S, et al. A novel ternary plasmonic photocatalyst: ultrathin $\mathrm{g}_{-} \mathrm{C}_{3} \mathrm{~N}_{4}$ nanosheet hybrided by $\mathrm{Ag} / \mathrm{AgVO}_{3}$ nanoribbons with enhanced visible-light photocatalytic performance. Appl Catal B Environ 2015, 165: 335-343.

8.Shi R, Ye HF, Liang F, et al. Interstitial P-doped CdS with long-lived photogenerated electrons for photocatalytic water splitting without sacrificial agents. Adv Mater 2018, 30: 1705941.

9.Zhang S, Li J, Wang X, et al. Rationally designed 1D $\mathrm{Ag} @ \mathrm{AgVO}_{3}$ nanowire/graphene /protonated $\mathrm{g}-\mathrm{C}_{3} \mathrm{~N}_{4}$ nanosheet heterojunctions for enhanced photocatalysis via electrostatic self-assembly and photochemical reduction methods. J Mater Chem A 2015, 3: 10119-10126.

10.Chen Y, Liang Y, Zhao M, et al. In situ ion exchange synthesis of $\mathrm{Ag}_{2} \mathrm{~S} / \mathrm{AgVO}_{3}$ graphene aerogels for enhancing photocatalytic antifouling efficiency. Ind Eng Chem Res 2019, 58: 3538-3548.

11.Huang CM, Pan GT, Li YCM, et al. Crystalline phases and photocatalytic activities of hydrothermal synthesis $\mathrm{Ag}_{3} \mathrm{VO}_{4}$ and $\mathrm{Ag}_{4} \mathrm{~V}_{2} \mathrm{O}_{7}$ under visible light irradiation. Appl Catal A Gen 2009, 358: 164-172.

12.Ran R, Meng X, Zhang Z. Facile preparation of novel graphene oxide-modified $\mathrm{Ag}_{2} \mathrm{O} / \mathrm{Ag}_{3} \mathrm{VO}_{4} / \mathrm{AgVO}_{3}$ composites with high photocatalytic activities under visible light irradiation. Appl Catal B Environ 2016, 196: 1-15.

13.Shen G, Chen D. Self-Coiling of $\mathrm{Ag}_{2} \mathrm{~V}_{4} \mathrm{O}_{11}$ Nanobelts into Perfect Nanorings and Microloops. J Am Chem Soc 2006, 128: 11762-11763.

14. Zhao W, Guo Y, Faiz Y, et al. Facile in-suit synthesis of $\mathrm{Ag} / \mathrm{AgVO}_{3}$ one-dimensional hybrid nanoribbons with enhanced performance of plasmonic visible-light photocatalysis. Appl Catal B Environ 2015, 163: 288-297.

15. Yang Y, Liu Y, Huang B, et al. Enhanced visible photocatalytic activity of a BiVO $\mathrm{B}_{4} @ \beta-\mathrm{AgVO}_{3}$ 
composite synthesized by an in situ growth method. RSC Adv 2014, 4: 20058-20061.

16.Sun M, Senthil RA, Pan J, et al. A Facile synthesis of visible-light driven Rod-on-Rod like $\alpha-\mathrm{FeOOH} / \alpha-\mathrm{AgVO}_{3}$ nanocomposite as greatly enhanced photocatalyst for degradation of Rhodamine B. Catalysts 2018, 8:392.

17.Chen LC, Teng CY, Lin CY, et al. Architecting nitrogen functionalities on graphene oxide photocatalysts for boosting hydrogen production in water decomposition process. Adv Energy Mater 2016, 6: 1600719.

18.Huang $\mathrm{Y}$ and Wan C. Controllable fabrication and multifunctional applications of graphene/ceramic composites. J Adv Ceram 2020, 9: 271-291.

19.Tian H, Liu M, Zheng W. Constructing 2D graphitic carbon nitride nanosheets/layered $\mathrm{MoS}_{2}$ /graphene ternary nanojunction with enhanced photocatalytic activity. Appl Catal B Environ 2018, 225: 468-476.

20.Clark SJ, Segall MD, Pickard CJ, et al. First principles methods using CASTEP. Z. Kristallogr 2005, 220: 567-570.

21.Segall MD, Lindan P, Probert MJ, et al. First-principles simulation: ideas, illustrations and the CASTEP code. J Phys: Condens Mater 2002, 14:2717-2744.

22.Pack JD, Monkhorst HJ. Special points for Brillouin-Zone inte-grations-A Reply. Phys Rev B 1977, 16:1748-1749.

23.Perdew JP, Burke K, Ernzerhof M, Generalized gradient approximation made simple. Phys Rev Lett 1996. 77: 3865-3868.

24.Zhou $\mathrm{Y}$ and $\mathrm{Xiang} \mathrm{H} . \mathrm{Al}_{5} \mathrm{BO}_{9}$ : A wide band gap, damage-tolerant, and thermal insulating lightweight material for high-temperature applications. J Am Chem Soc 2016, 99:2742-2751.

[25] J. Donnay and D. Harker, A new law of crystal morphology extending the Law of Bravais, Am Mineral 1937, 22: 457-477.

[26] A. F. Wells, XXI. Crystal habit and internal structure.-I, The London, Edinburgh, and Dublin Philosophical Magazine and Journal of Science 1946, 37:184-199.

[27] Z. Berkovitch-Yellin, Toward an ab initio derivation of crystal morphology, J.Am.Chem.Soc 1985, 107: 8239-8253.

28.Sivakumar V, Suresh R, Giribabu K, et al. $\mathrm{AgVO}_{3}$ nanorods: Synthesis, characterization and visible light photocatalytic activity. Solid State Sci 2015, 39: 34-39.

29.Barebita H, Ferraa S, Moutataouia M, et al. Structural investigation of $\mathrm{Bi}_{2} \mathrm{O}_{3}-\mathrm{P}_{2} \mathrm{O}_{5}-\mathrm{B}_{2} \mathrm{O}_{3}-\mathrm{V}_{2} \mathrm{O}_{5}$ quaternary glass system by Raman, FTIR and thermal analysis. Chem Phys Lett 2020, 760: 138031.

30.Li Q, Guo B, Yu J, et al. Highly Efficient Visible-light-driven photocatalytic hydrogen production of CdS-cluster-decorated graphene nanosheets. J Am Chem Soc 2011, 133: 10878-10884.

31.Wang H, Robinson JT, Li X, et al. Solvothermal reduction of chemically exfoliated graphene sheets. J Am Chem Soc 2009, 131: 9910-9911. 
32.Zhao $\mathrm{W}$, Li J, Wei $\mathrm{Z} \mathrm{b}$, et al. Fabrication of a ternary plasmonic photocatalyst of $\mathrm{Ag} / \mathrm{AgVO}_{3} / \mathrm{RGO}$ and its excellent visible-light photocatalytic activity. Appl Catal B Environ 2015, 179: 9-20.

33.Kong X, Guo Z, Zeng C, et al. Soft chemical in situ synthesis, formation mechanism and electrochemical performances of 1D bead-like $\mathrm{AgVO}_{3}$ nanoarchitectures. J Mater Chem A 2015, 3: 18127-18135.

34.Oliveira RC, Foggi CC, Teixeira MM, et al. Mechanism of antibacterial activity via morphology change of $\alpha-\mathrm{AgVO}_{3}$ : Theoretical and experimental insights. ACS Appl Mater Interfaces 2017, 9: 11472-11481.

35.Song JM, Lin YZ, Yao HB, et al. Superlong $\beta-\mathrm{AgVO}_{3}$ nanoribbons: High-yield synthesis by a pyridine-assisted solution approach, their Stability, electrical and electrochemical properties. ACS Nano 2009, 3: 653-660.

36.Wang $\mathrm{F}$, $\mathrm{Li} \mathrm{F}$, Zhang $\mathrm{L}$, et al. $\mathrm{S}-\mathrm{TiO}_{2}$ with enhanced visible-light photocatalytic activity derived from $\mathrm{TiS}_{2}$ in deionized water. Mater Res Bull 2017, 87: 20-26.

37.Gao L, Li Z, Liu J. Facile synthesis of $\mathrm{Ag}_{3} \mathrm{VO}_{4} / \beta-\mathrm{AgVO}_{3}$ nanowires with efficient visible-light photocatalytic activity. $R S C A d v$ 2017, 7: 27515-27521.

38.Wang $R$ and $\mathrm{Cao} \mathrm{L}$. Facile synthesis of a novel visible-light-driven $\mathrm{AgVO}_{3} / \mathrm{BiVO}_{4}$ heterojunction photocatalyst and mechanism insight. J Alloys Compd 2017, 722: 445-451.

39.Zhang J, Wang J, Xu H, et al. The effective photocatalysis and antibacterial properties of $\mathrm{AgBr} / \mathrm{AgVO}_{3}$ composites under visible-light. $R S C$ Adv 2019, 9: 37109-37118.

40. Wang J, Yang X, Chen J, et al. Photocatalytic activity of novel $\mathrm{Ag}_{4} \mathrm{~V}_{2} \mathrm{O}_{7}$ photocatalyst under visible light irradiation. J Am Ceram Soc 2014, 97: 267-274.

41. Zhang T, Zhao D, Wang Y, et al. Facial synthesis of a novel $\mathrm{Ag}_{4} \mathrm{~V}_{2} \mathrm{O}_{7} / \mathrm{g}-\mathrm{C}_{3} \mathrm{~N}_{4}$ heterostructure with highly efficient photoactivity. J Am Ceram Soc 2019,102:3897-3907.

42. Zhang L, He Y, Ye P, et al. Enhanced photodegradation activity of Rhodamine B by $\mathrm{Co}_{3} \mathrm{O}_{4} / \mathrm{Ag}_{3} \mathrm{VO}_{4}$ under visible light irriadiation. Mat Sci Eng B 2013, 178: 45-52.

43.Perdew JP, Ruzsinszky A, Csonka GI, et al. Restoring the density-gradient expansion for exchange in solids and surfaces. Phys Rev Lett 2008, 100: 136406.

44.Heyd J, Scuseria GE, Ernzerhof M. Hybrid functionals based on a screened Coulomb potential. J Chem Phys 2003, 118: 8207-8215.

45.Tandon SP and Gupta JP. Measurement of forbidden energy gap of semiconductors by diffuse reflectance technique. Phys Status Solidi B 1970, 38: 363-367.

46.Liu J, Liu Y, Liu N, et al. Metal-free efficient photocatalyst for stable visible water splitting via a two-electron pathway. Science 2015, 347: 970.

47.Wu X, Zhao J, Wang L, et al. Carbon dots as solid-state electron mediator for $\mathrm{BiVO}_{4} / \mathrm{CDs} / \mathrm{CdS}$ Z-scheme photocatalyst working under visible light. Appl Catal B Environ 2017, 206: 501-509.

48.Zheng Y, Yu Z, Ou H, et al. Black phosphorus and polymeric carbon nitride heterojunction for photoinduced molecular oxygen activation. Adv Funct Mater 2018, 28: 1705407. 
49.Luo G, Jiang X, Li M, et al. Facile fabrication and enhanced photocatalytic performance of $\mathrm{Ag} / \mathrm{AgCl} / \mathrm{rGO}$ heterojunction photocatalyst. ACS Appl Mater Inter 2013, 5: 2161-2168.

50.Liu D, Chen D, Li N, et al. Integration of 3D macroscopic graphene aerogel with 0D-2D $\mathrm{AgVO}_{3}-\mathrm{g}-\mathrm{C}_{3} \mathrm{~N}_{4}$ heterojunction for highly efficient photocatalytic oxidation of nitric oxide. Appl Catal B Environ 2019, 243: 576-584.

51.Ran R, McEvoy JG, Zhang Z. $\mathrm{Ag}_{2} \mathrm{O} / \mathrm{Ag}_{3} \mathrm{VO}_{4} / \mathrm{Ag}_{4} \mathrm{~V}_{2} \mathrm{O}_{7}$ heterogeneous photocatalyst prepared by a facile hydrothermal synthesis with enhanced photocatalytic performance under visible light irradiation. Mater Res Bull 2016, 74: 140-150.

52.Cao S, Liu T, Tsang Y, et al. Role of hydroxylation modification on the structure and property of reduced graphene oxide/TiO 2 hybrids. Appl Surf Sci 2016, 382: 225-238. 
Figures
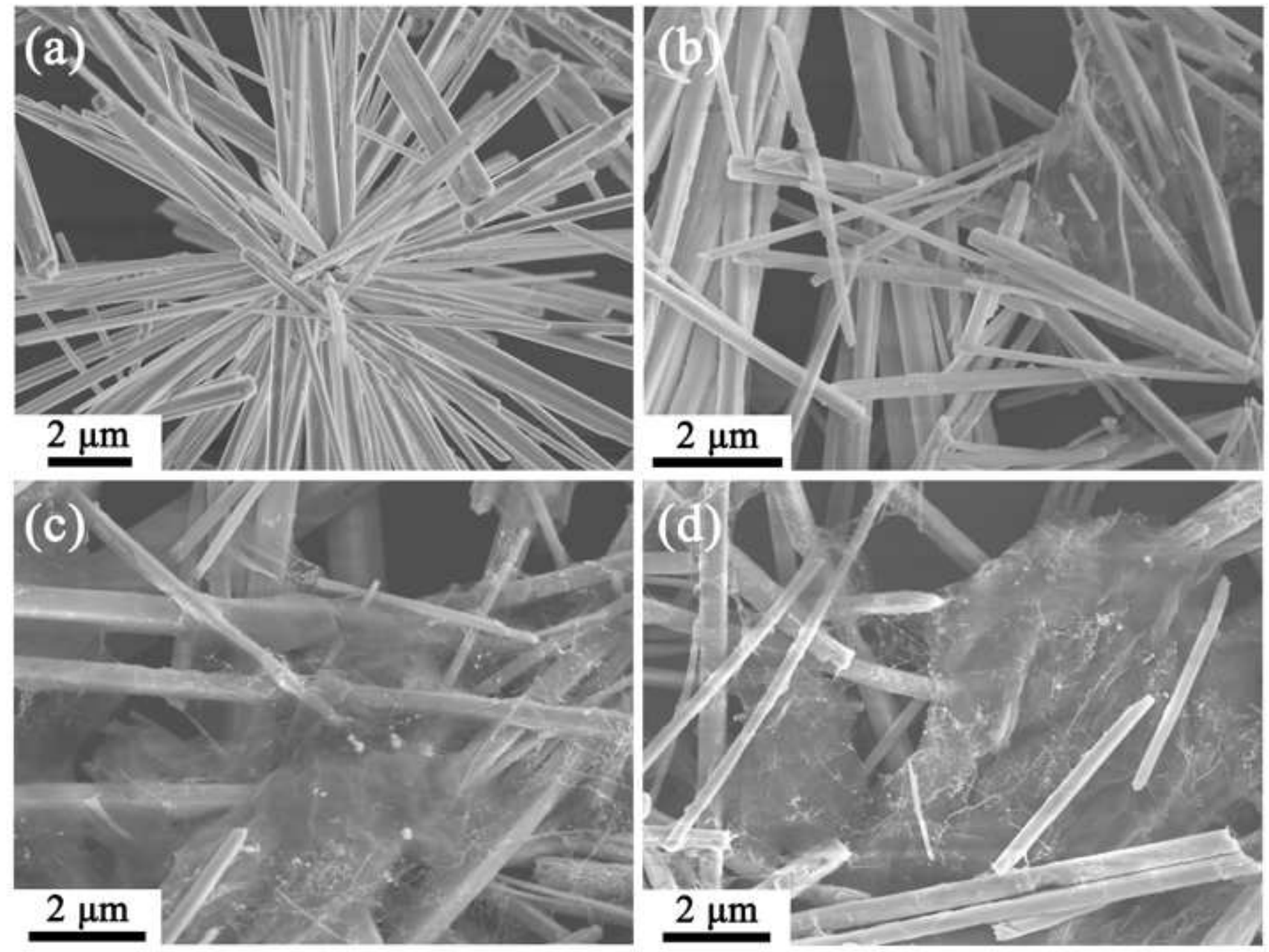

\section{Figure 1}

SEM images of the as-prepared (a) a-AgVO3, (b) $0.5 \mathrm{GO} / \mathrm{a}-\mathrm{AgVO} 3$, (c) $1.0 \mathrm{GO} / \mathrm{a}-\mathrm{AgVO} 3$, (d) $2.0 \mathrm{GO} / \mathrm{a}-$ AgV03. 


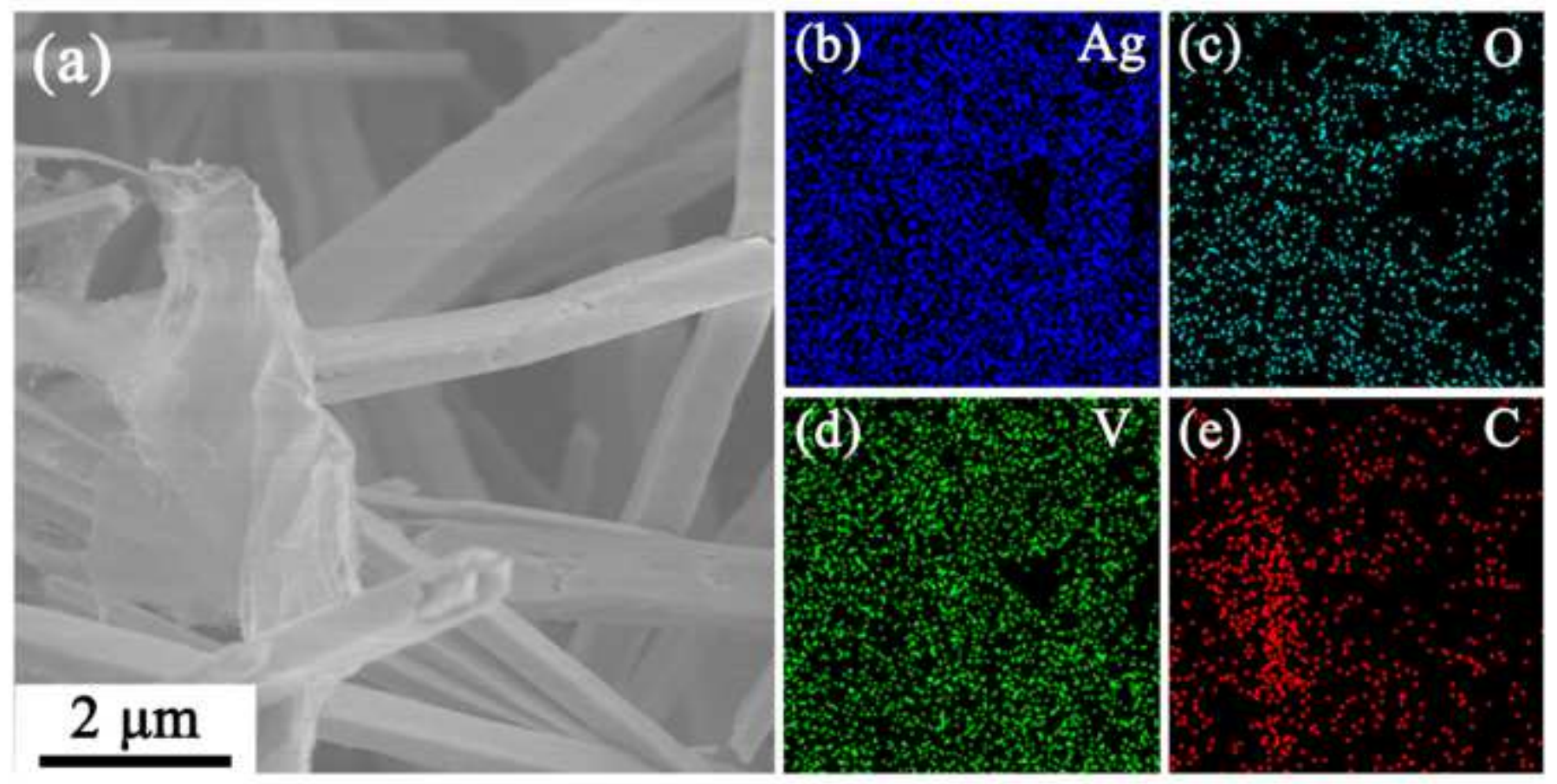

Figure 2

The SEM image of $0.5 \mathrm{GO} / \mathrm{a}-\mathrm{AgVO} 3$ (a) and the corresponding EDS elemental mapping of (b) Ag, (c) 0 , (d) V, and (e) C.

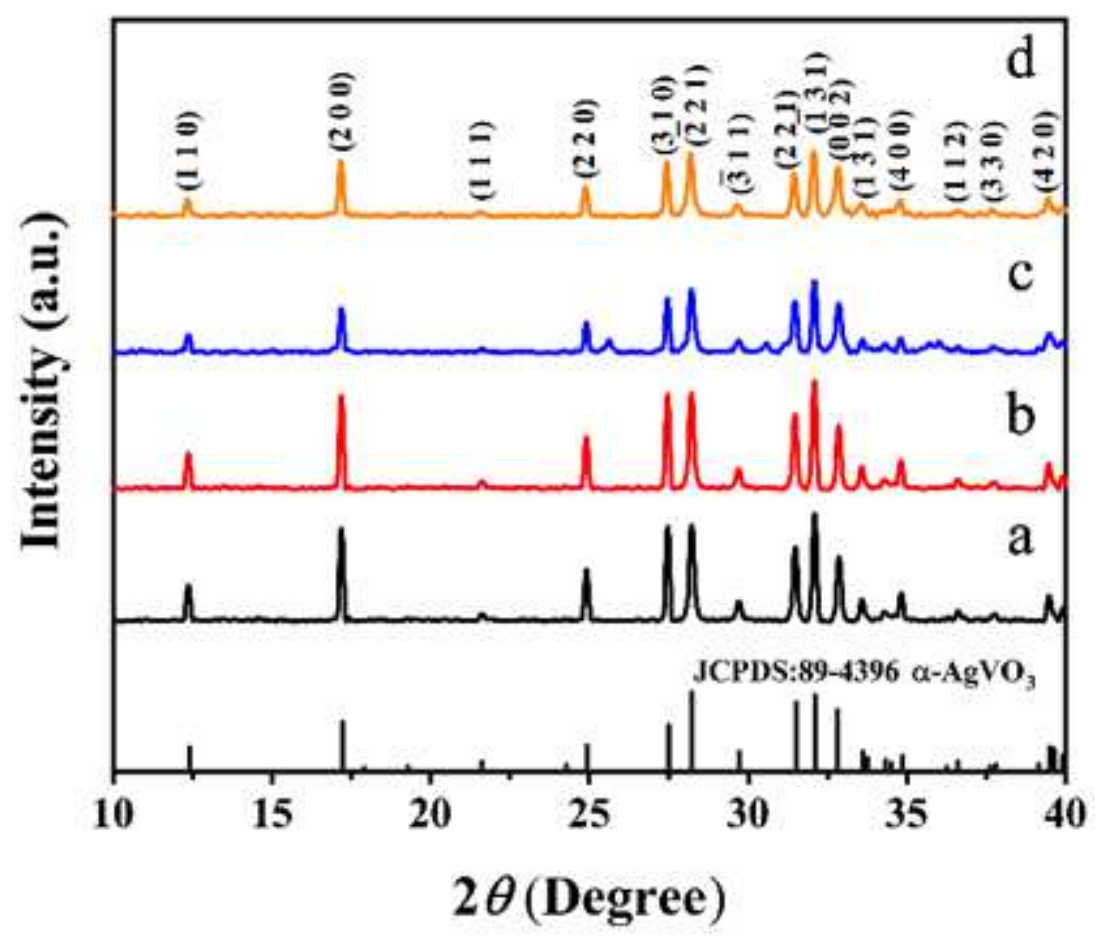

Figure 3

XRD patterns of (a) a-AgVO3, (b) 0.5 GO/a-AgVO3, (c) $1.0 \mathrm{GO} / \mathrm{a}-\mathrm{AgVO} 3$ and (d) $2.0 \mathrm{GO} / \mathrm{a}-\mathrm{AgVO} 3$. 


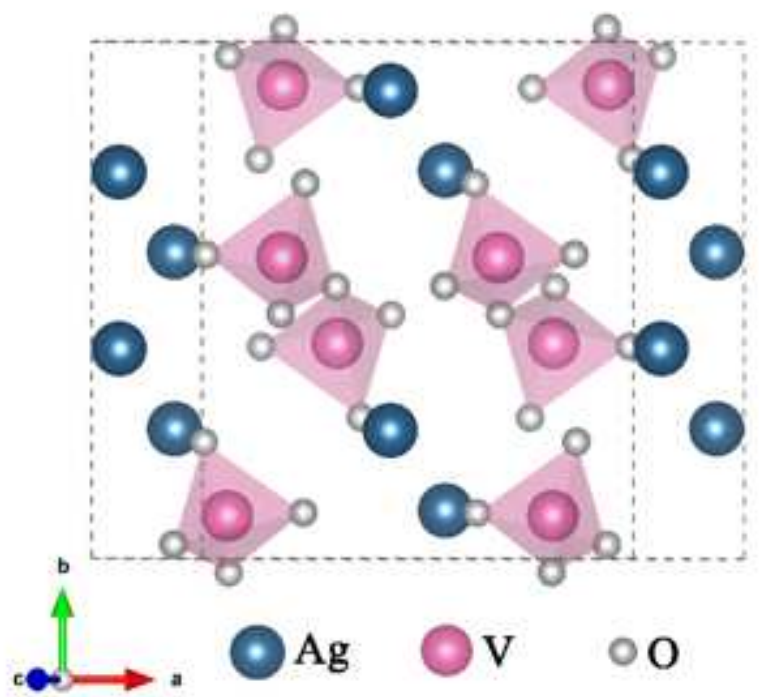

Figure 4

Crystal structure of a-AgVO3.
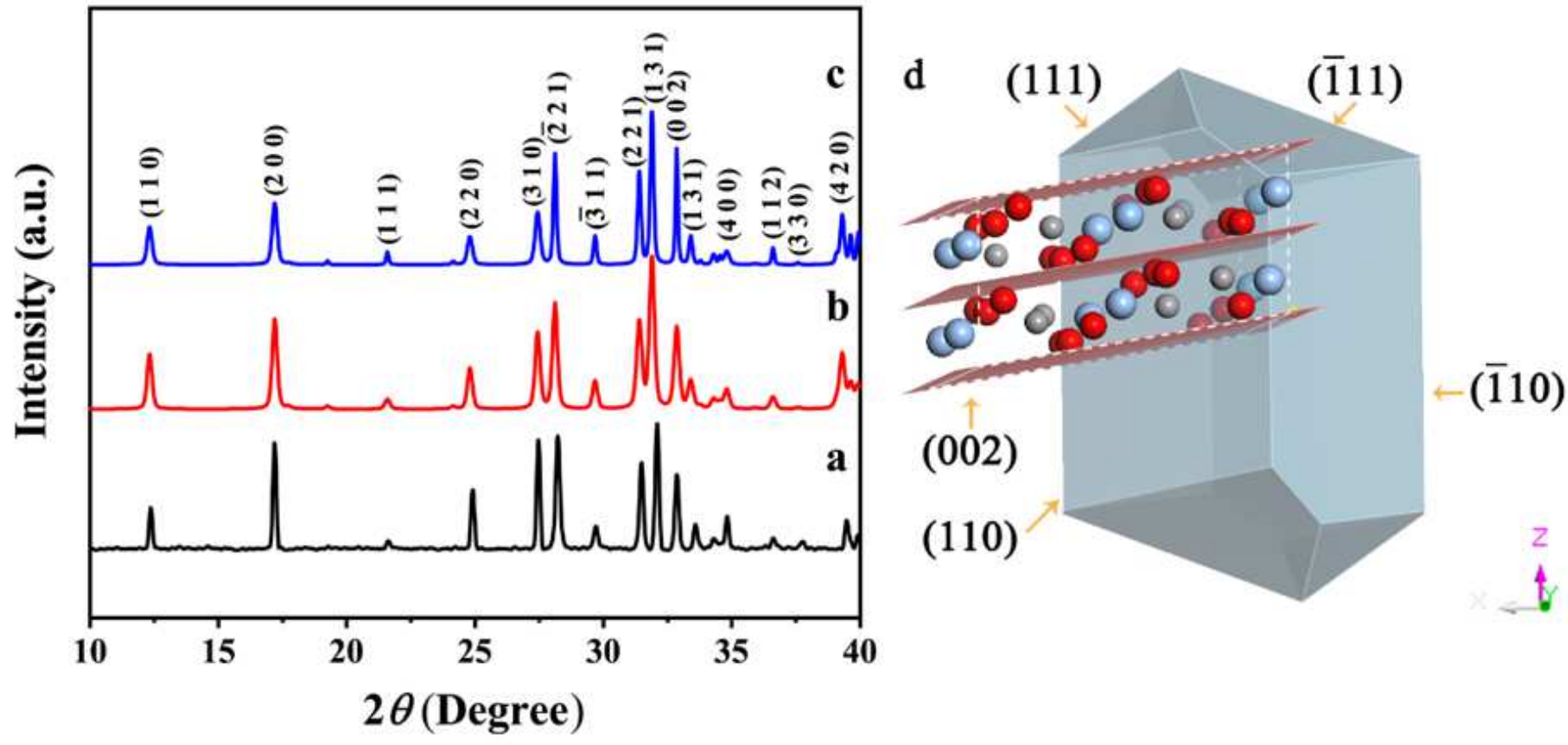

Figure 5

Experimental XRD patterns of a-AgVO3 (a), together with simulated XRD patterns of powder (b) and caxis oriented particle (c). (d) the calculated morphology based on the theory of Donnay-Harker. (The purple crystal is the a-AgVO3 nanorod, and the plane is the $\left(\begin{array}{lll}0 & 0\end{array}\right)$ plane.) 

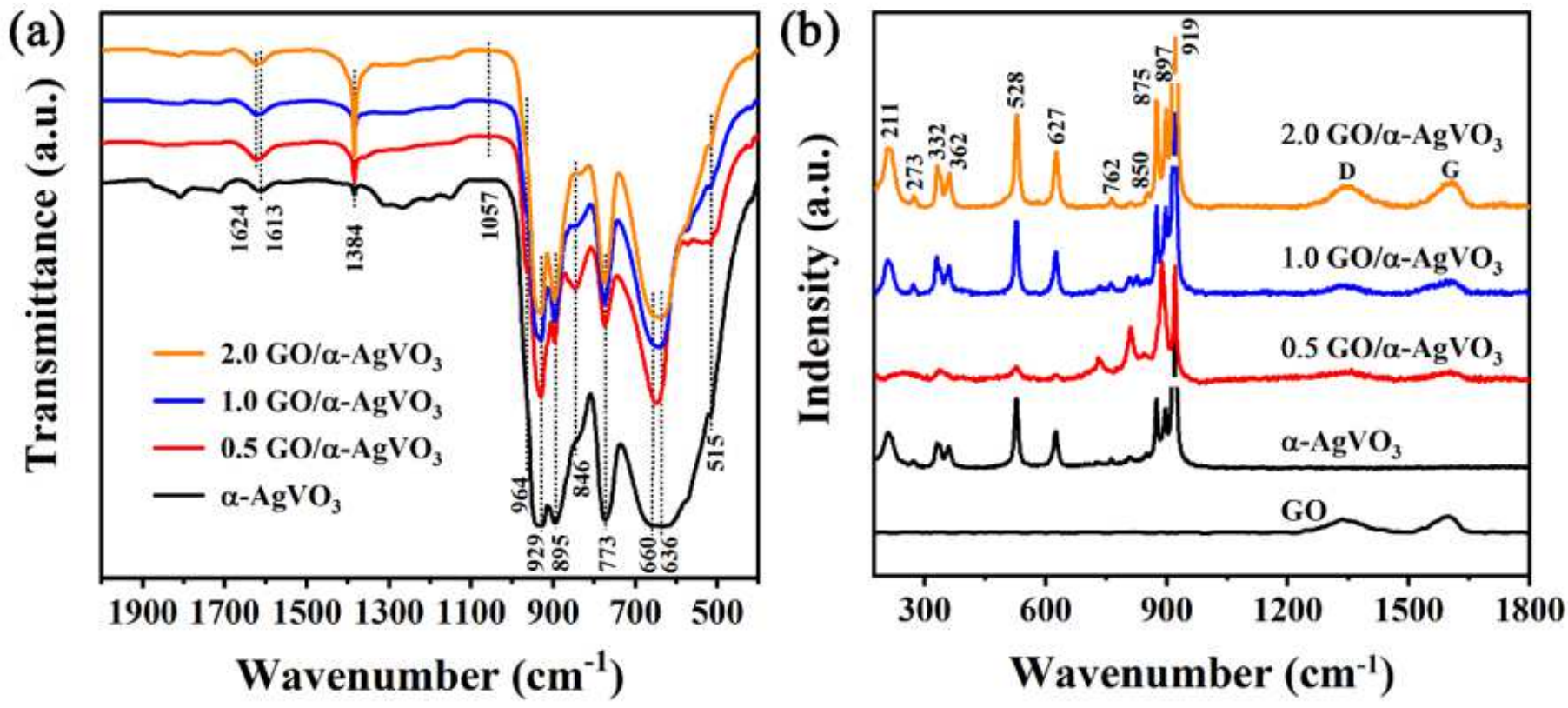

Figure 6

(a) FTIR spectra and (b) Raman spectra of a-AgVO3 and GO/a-AgVO3 with different GO contents (in 0.5 wt.\%, 1.0 wt.\%, and 2.0 wt.\%).
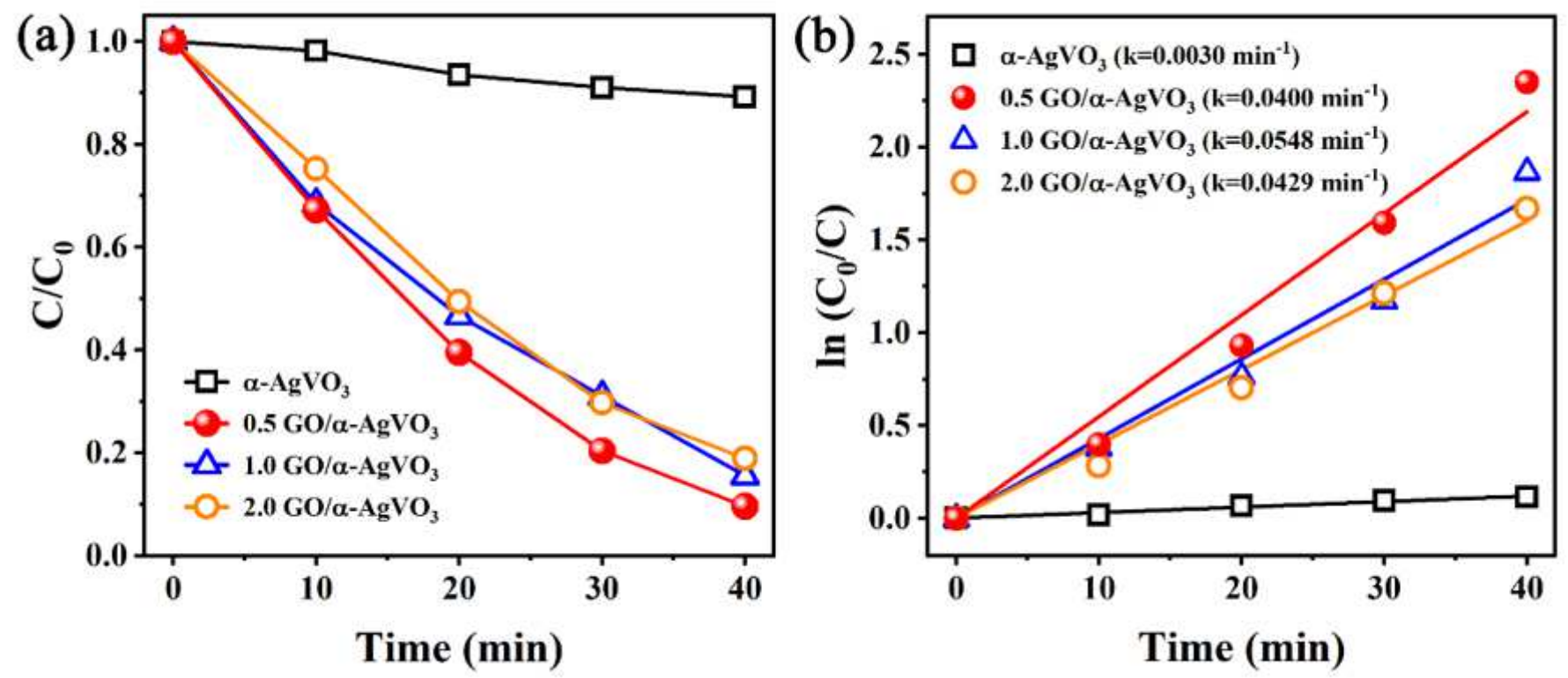

Figure 7

Photocatalytic degradation curves of RhB (a) and the corresponding pseudo-first-order kinetic curves (b). 

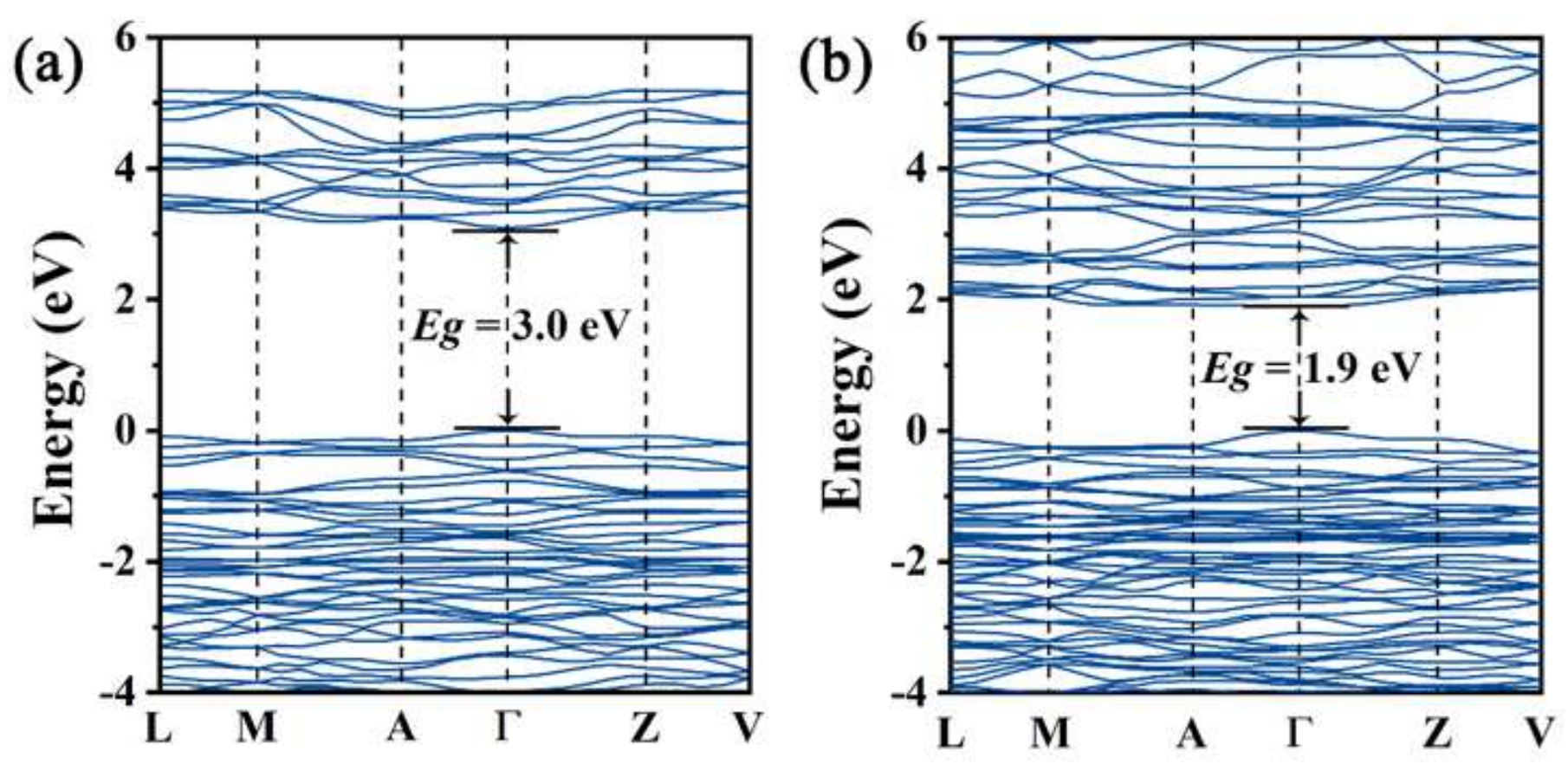

Figure 8

Band structure of a-AgVO3 along the high symmetry line in the Brillouin zone calculated by (a) GGA and (b) HSE03 functional.
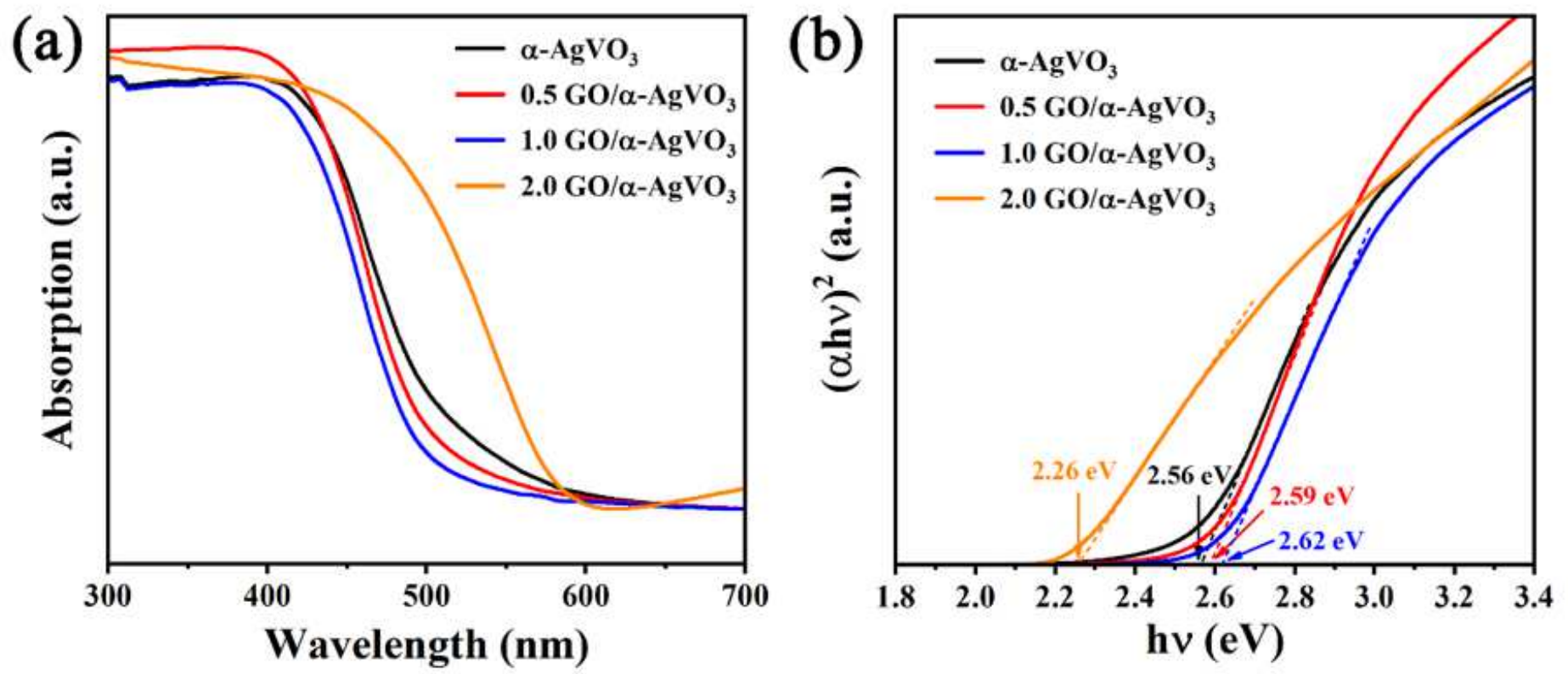

Figure 9

UV-vis diffuse reflectance spectra (a) and Kubelka-Munk plot (b) of a-AgV03 and G0/a-AgV03 with different GO contents (in 0.5 wt.\%, 1.0 wt.\%, and 2.0 wt.\%). 

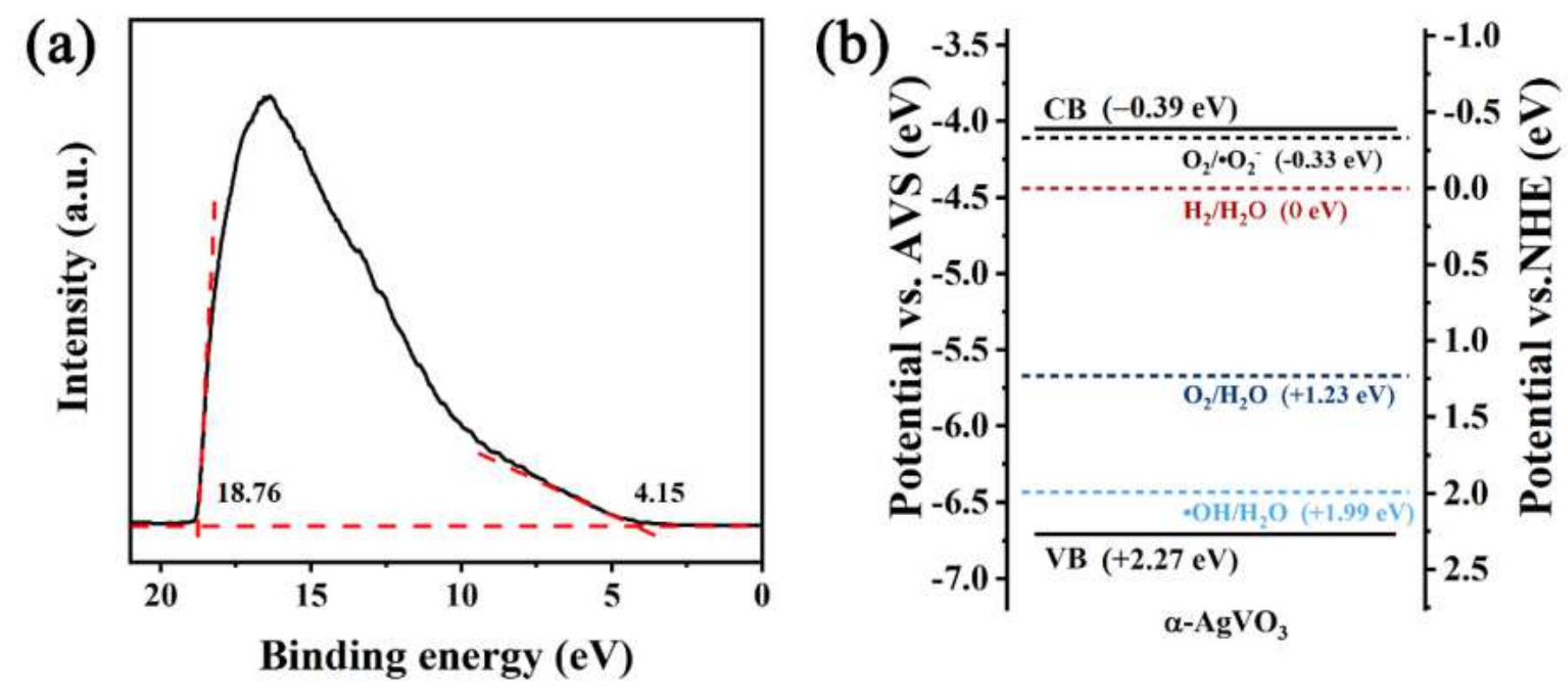

Figure 10

UPS spectra (a) and the band structure diagram of a-AgVO3(b). 

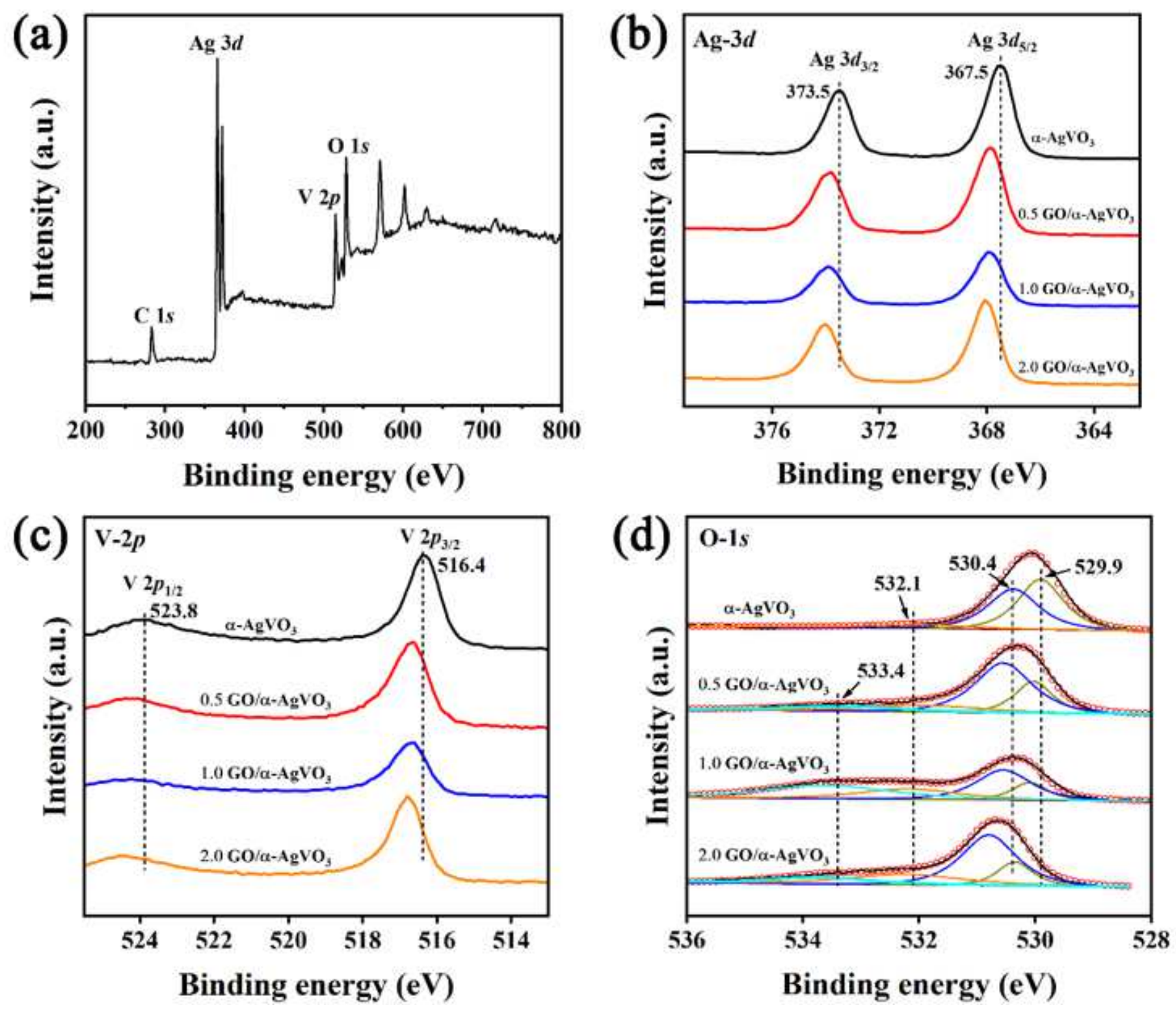

Figure 11

Full-scale XPS survey spectrum for $0.5 \mathrm{GO} / \mathrm{a}-\mathrm{AgVO} 3$ (a) and the XPS spectra of (b) Ag 3d, (c) $\vee 2 p$ and 0 1s for GO/a-AgVO3 with different GO contents (in 0.5 wt.\%, 1.0 wt.\%, and 2.0 wt.\%). 


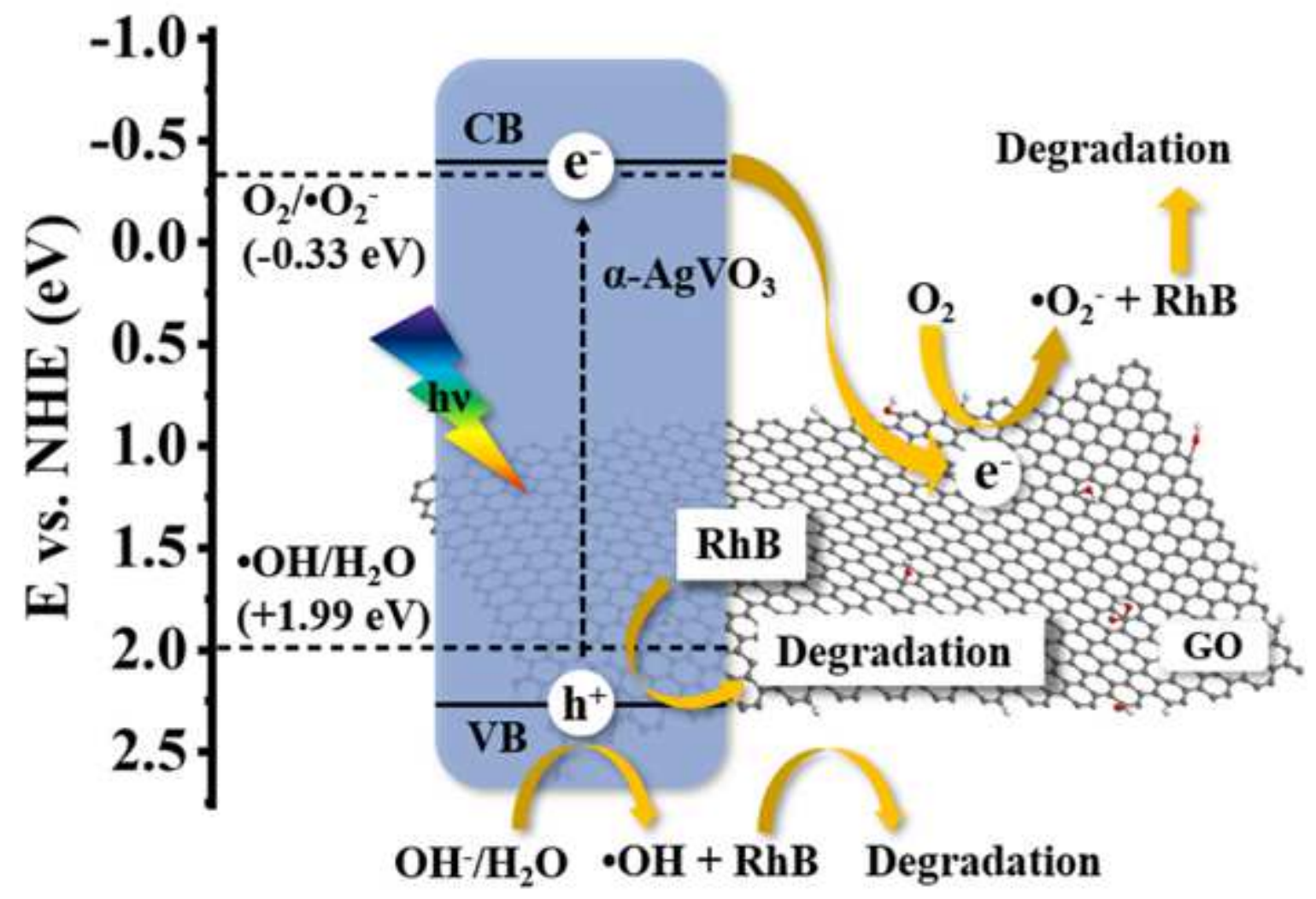

Figure 12

Proposed photocatalytic mechanism of GO/a-AgVO3 photocatalyst. 